\title{
MULTIPLICITY OF THE GALACTIC SENIOR CITIZENS: A HIGH-RESOLUTION SEARCH FOR COOL SUBDWARF COMPANIONS
}

\author{
Carl Ziegler ${ }^{1}$, Nicholas M. LaW ${ }^{1}$, Christoph Baranec ${ }^{2}$, Reed L. Riddle ${ }^{3}$, and Joshua T. Fuchs ${ }^{1}$ \\ ${ }^{1}$ Department of Physics and Astronomy, University of North Carolina at Chapel Hill, Chapel Hill, NC 27599-3255, USA; carlziegler@unc.edu \\ ${ }^{2}$ Institute for Astronomy, University of Hawai' $i$ at Mānoa, HI 96720-2700, USA \\ ${ }^{3}$ Division of Physics, Mathematics, and Astronomy, California Institute of Technology, Pasadena, CA 91125, USA \\ Received 2014 November 11; accepted 2015 February 16; published 2015 April 27
}

\begin{abstract}
Cool subdwarfs are the oldest members of the low-mass stellar population. Mostly present in the galactic halo, subdwarfs are characterized by their low-metallicity. Measuring their binary fraction and comparing it to solarmetallicity stars could give key insights into the star formation process early in the Milky Way's history. However, because of their low luminosity and relative rarity in the solar neighborhood, binarity surveys of cool subdwarfs have suffered from small sample sizes and incompleteness. Previous surveys have suggested that the binary fraction of red subdwarfs is much lower than for their main-sequence cousins. Using the highly efficient Robo-AO system, we present the largest high-resolution survey of subdwarfs, sensitive to angular separations $(\rho \geqslant 0$ "' 15$)$ and contrast ratios $\left(\Delta m_{i} \leqslant 6\right)$ invisible in past surveys. Of 344 target cool subdwarfs, 43 are in multiple systems, 19 of which are newly discovered, for a binary fraction of $12.5 \pm 1.9 \%$. We also discovered seven triple star systems for a triplet fraction of $2.0 \pm 0.8 \%$. Comparisons to similar surveys of solar-metallicity dwarf stars gives a $\sim 3 \sigma$ disparity in luminosity between companion stars, with subdwarfs displaying a shortage of low-contrast companions. We also observe a lack of close subdwarf companions in comparison to similar-mass dwarf multiple systems.
\end{abstract}

Key words: binaries: close - instrumentation: adaptive optics - methods: data analysis - stars: late-type subdwarfs - techniques: high angular resolution

\section{INTRODUCTION}

Cool subdwarfs are the oldest members of the low-mass stellar population, with spectral types of $\mathrm{G}, \mathrm{K}$, and $\mathrm{M}$, masses between $\sim 0.6$ and $\sim 0.08 M_{\odot}$, and surface effective temperatures between 4000 and $2300 \mathrm{~K}$ (Kaltenegger \& Traub 2009). First coined by Kuiper (1939), subdwarfs are the lowluminosity, metal-poor $([\mathrm{Fe} / \mathrm{H}]<-1)$ spectral counterparts to the main sequence dwarfs. On a color-magnitude diagram, subdwarfs lie between white dwarfs and the main sequence (Adams 1915). With decreased metal opacity, subdwarfs have smaller stellar radii and are bluer at a given luminosity than their main sequence counterparts (Sandage \& Eggen 1959). These low-mass stars are members of the Galactic halo (Gould 2003) and have higher systematic velocities and proper motions than disk dwarf stars. Traditionally subdwarfs have been identified using high proper motion surveys. Although $99.7 \%$ of stars in the galaxy are disk main sequence, statistically there are more subdwarfs in these high PM surveys (Reid \& Hawley 2005). Verification and precise spectral typing of cool subdwarfs can be performed by measuring molecular lines, as defined first by Gizis (1997). Lépine et al. (2007) introduced a refined system, using spectroscopic measurements of a large 1983 star survey to standardize the subdwarf metallicity subclasses and spectroscopic sequence.

The search for companions to stars of different masses gives clues to the star formation process, as any successful model must account for both the frequency of the multiple star systems and the properties of the systems. In addition, monitoring the orbital characteristics of multiple star systems yields information otherwise unattainable for single stars, such as relative brightness and masses of the components (Goodwin et al. 2007), lending further constraints to mass-luminosity relationships (Chabrier et al. 2000).
Old population II stars are important probes for the early history of star formation in the galaxy (Zhang et al. 2013). The formation process of low-mass stars remains less well understood than for solar-like stars. Although multiple indications suggest they form as the low-mass tail of regular star formation (Bourke et al. 2006), other mechanisms have been proposed for some or all of these objects (Goodwin \& Whitworth 2007; Thies \& Kroupa 2007; Basu \& Vorobyov 2012). A firm binary fraction for low-metallicity cool stars could assist in constraining various formation models. This again motivates the need for a comprehensive binarity survey, sensitive to small angular separations.

The multiplicity of main sequence dwarfs has been well explored in the literature. A consistent trend that has purveyed is that the percentage of stars with stellar companions seems to depend on the mass of the stars. For AB-type stars, Peter et al. (2012) used a sample of 148 stars to determine a companion fraction of $\sim 70 \%$. For solar type stars (FGK-type), around 57\% have companions (Duquennoy \& Mayor 1991), although Raghavan et al. (2010) have revised the fraction down to $\sim 46 \%$. Fischer \& Marcy (1992) looked at M-dwarfs and found a multiplicity fraction of $42 \pm 9 \%$. More recently, Janson et al. (2012) found a binary fraction for late K- to mid M-type dwarfs of $27 \pm 3 \%$ from a sample of 701 stars. For late Mdwarfs, a slightly lower fraction was found by Law et al. (2006b) of $7 \% \pm 3 \%$. Extending their previous study for mid/ late M-type dwarfs, M5-M8, Janson et al. (2014) find a multiplicity fraction of $21 \%-27 \%$ using a sample of 205 stars.

While the multiplicity of dwarf stars has been heavily studied with comprehensive surveys, detailed multiplicity studies of low-mass subdwarfs have, historically, been hindered by their low luminosities and relative rarity in the solar neighborhood. Within $10 \mathrm{pc}$, there are three low-mass subdwarfs, compared to 
243 main sequence stars (Monteiro et al. 2006). Subsequently, multiplicity surveys of cool subdwarfs have been relatively small. The largest, a low-limit angular resolution search by Zhang et al. (2013) mined the Sloan Digital Sky Survey (SDSS; York et al. 2000) to find 1826 cool subdwarfs, picking out subdwarfs by their PMs and identifying spectral type by fitting an absolute magnitude-spectral type relationship. They find 45 subdwarfs multiple systems in total, with 30 being wide companions and 15 partially resolved companions. When adjusting for the incompleteness of their survey, an estimate of the binary fraction of $>10 \%$ is predicted. The authors note the need for a high spatial resolution imaging survey to search for close binaries $(<100 \mathrm{AU})$ and put tighter constraints on the binary fraction of cool subdwarfs.

The high-resolution subdwarf surveys completed thus far have been comparatively small. Gizis \& Reid (2000) detected no companions in a sample of eleven cool subdwarfs. Riaz et al. (2008) similarly found no companions in a sample of 19 M-subdwarfs using the Hubble Space Telescope. Lodieu et al. (2009) reported one companion in a sample of $33 \mathrm{M}$ type subdwarfs. Jao et al. (2009) found four companions in a sample of 62 cool subdwarf systems. With the high variance in small number statistics, the relationship between dwarf and subdwarf multiplicity fractions remains inconclusive.

We present here the largest high resolution cool subdwarf multiplicity survey yet performed, making use of the efficient Robo-AO system (Baranec et al. 2014). The Robo-AO system allows us to detect more cool and close companion stars in a much larger sample size than previously possible. This survey combines previously known wide proper-motion pairs, spectroscopic binaries, and high angular resolution images able to detect companions with $\rho \geqslant 0$ ". 15 and $\Delta m_{i} \leqslant 6$.

The paper is organized as follows. In Section 2 we describe the target selection, the Robo-AO system, and follow-up observations. In Section 3 we describe the Robo-AO data reduction and the companion detection and analysis. In Section 4 we describe the results of this survey, including discovered companions, and compare to similar dwarf surveys. The results are discussed in Section 5 and put in context of previous literature. We conclude in Section 6.

\section{SURVEY TARGETS AND OBSERVATIONS}

\subsection{Sample Selection}

We selected targets from the 564 spectral type F- through Msubdwarf candidates studied by Marshall (2007). These targets were selected from the New Luyten Two-Tenths catalog (NLTT; Luyten 1979; Luyten \& Hughes 1980) of high proper

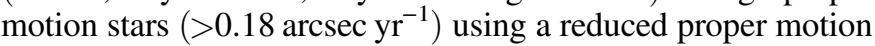
diagram (RPM). To distinguish subdwarf stars from their solarmetallicity companions on the main sequence, the RPM used a $(V-J)$ optical-infrared baseline, a technique first used by Salim \& Gould (2002), rather than the shorter $(B-R)$ baseline used by Luyten. This method uses the high proper motion as a proxy for distance and the blueness of subdwarfs relative to equal luminosity dwarf stars to separate out main sequence members of the local disk and the halo subdwarfs (Marshall 2008). The RPM, $\mathrm{H}_{M}$, is defined as

$$
H_{M}=m+5 \log \mu+5
$$

where $m$ is the apparent magnitude and $\mu$ is the proper motion in "/yr. The discriminator, $\eta$, developed by Salim and Gould to

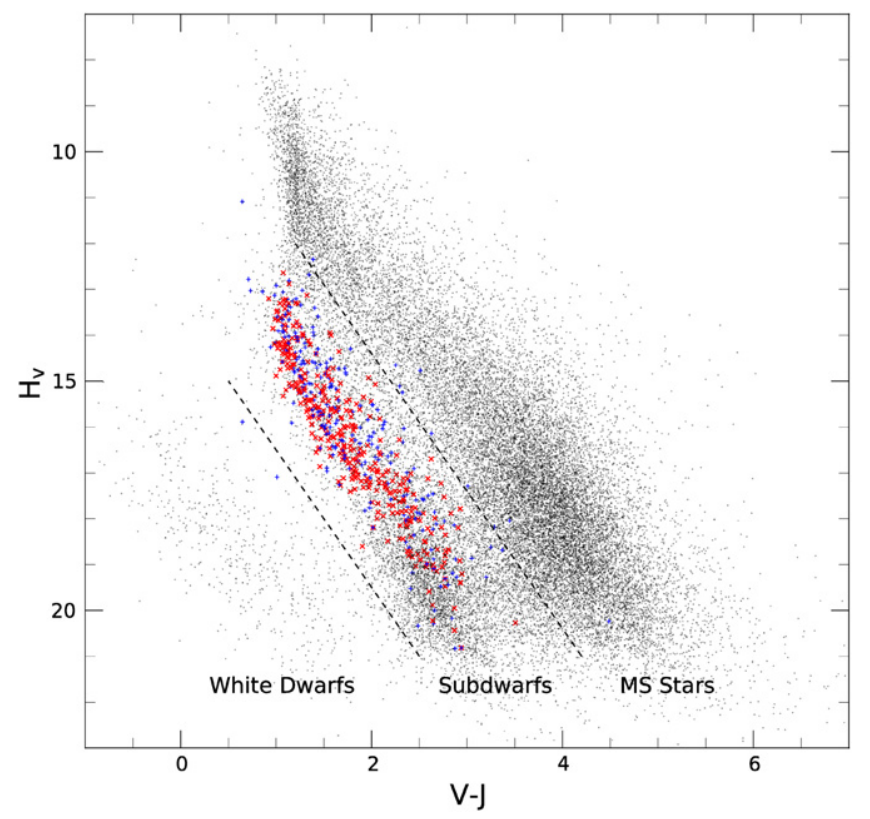

Figure 1. Reduced proper motion diagram of the complete rNLTT (Gould \& Salim 2003), with our observed subdwarfs in red $X$ 's, drawn from the photometric work of Marshall (2007). Unobserved candidate subdwarfs from Marshall (2007) are plotted as blue +'s. The discriminator lines, described in Section 2.1, between solar-metallicity dwarfs, metal-poor subdwarfs, and white dwarfs are at $\eta=0$ and 5.15, respectively, and with $b= \pm 30$. The subdwarfs plotted make use of the improved photometry of Marshall (2007).

separate luminosity classes, is defined as

$$
\begin{aligned}
\eta\left(H_{V}, V-J, \sin b\right)= & H_{V}-3.1(V-J) \\
& -1.47|\sin b|-7.73
\end{aligned}
$$

where $b$ is the Galactic latitude. The RPM diagram for the revised NLTT (rNLTT) catalog (Gould \& Salim 2003) and our subdwarf targets is presented in Figure 1. The improved photometry of Marshall (2007) placed 12 of the original suspected subdwarfs outside the subdwarf sequence. These stars were not included in our sample. Possible dwarf contamination of our sample is expected to be small, as described in Section 3.3. Of the 552 subdwarfs confirmed by Marshall, a randomly selected sample of 348 G-, K-, and M-subdwarfs were observed by Robo-AO when available between other high priority surveys. The $V$-band magnitudes and $(V-J)$ colors of the observed subdwarf sample are shown in Figure 2.

\subsection{Observations}

\subsubsection{Robo-AO}

We obtained high-angular-resolution images of the 348 subdwarfs during 32 separate nights of observations between 2012 September 3 and 2013 August 21 (UT). The observations were performed using the Robo-AO laser adaptive optics system (Riddle et al. 2012; Baranec et al. 2013, 2014) mounted on the Palomar 60 inch telescope. The first robotic laser guide star adaptive optics system, the automatic Robo-AO system can efficiently observe large, high-resolution surveys. All images were taken using the Sloan $i$-band filter (York et al. 2000) and with exposure times of $120 \mathrm{~s}$. Typical seeing at the Palomar Observatory is between 0 ". 8 and 1 ". 8 , with the median around 1."1 (Baranec et al. 2014). The typical FWHM (diffraction 

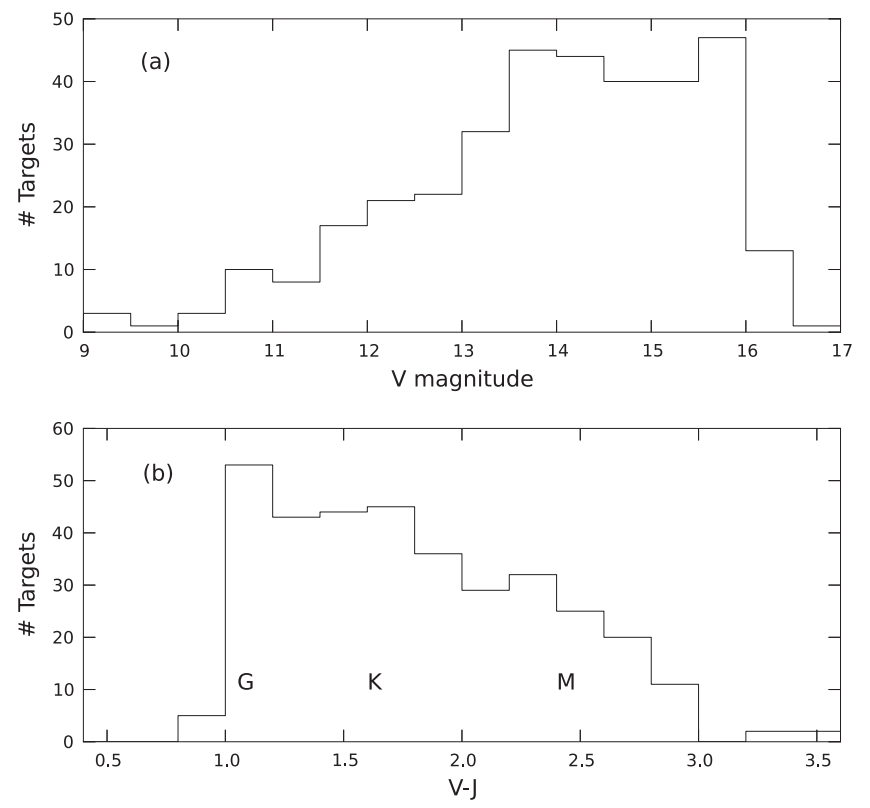

Figure 2. (a) Histogram of magnitudes in $V$-band of the 348 observed subdwarfs. (b) Histogram of the $(V-J)$ colors of the observed subdwarf sample, with approximate spectral types regions $\mathrm{G}, \mathrm{K}$, and $\mathrm{M}$ marked, using the spectral color indices of Ducati et al. (2001). Both plots use the photometry of Marshall (2007).

Table 1

The Specifications of the Robo-AO Subdwarf Survey

\begin{tabular}{ll}
\hline \hline Filter & Sloan $i^{\prime}$-band \\
\hline FWHM resolution & $00^{\prime \prime} 15$ \\
Field size & $44^{\prime \prime} \times 44^{\prime \prime}$ \\
Detector format & $1024^{2}$ pixels \\
Pixel scale & $43.1 \mathrm{mas} / \mathrm{pix}$ \\
Exposure time & $120 \mathrm{~s}$ \\
Subdwarf targets & 344 \\
Targets observed/hour & 20 \\
Observation dates & $2012 \mathrm{Sep} \mathrm{1-}$ \\
& 2013 Aug 21 \\
\hline
\end{tabular}

limited) resolution of the Robo-AO system is 0 ". $12-0$ ". 15 . Images are recorded on an electron-multiplying CCD (EMCCD), allowing short frame rates for tip and tilt correction in software using a natural guide star $\left(m_{V}<16\right)$ in the field of view (FOV). Specifications of the Robo-AO system are summarized in Table 1.

The images were reduced by the Robo-AO imaging pipeline described in Law et al. (2009, 2014). The EMCCD output frames are dark-subtracted and flat-fielded and then, using the Drizzle algorithm (Fruchter \& Hook 2002), stacked and aligned, while correcting for image motion using a star in the field. The algorithm also introduces a factor of 2 up-sampling to the images. Since the subdwarf targets are in relatively sparse stellar fields, for the majority of the images the only star visible is the target star and it was thus used to correct for the image motion.

\subsubsection{Keck $L G S-A O$}

Six candidate multiple systems were selected for re-imaging by the NIRC2 camera behind the Keck II laser guide star adaptive optics system (van Dam et al. 2006; Wizinowich et al.
2006), on 2014 August 17 (UT) to confirm possible companions. The targets were selected for their low significance of detectability, either because of low contrast ratio or small angular separation. The observations were done in the $K^{\prime}$ and $H$-bands with three $90 \mathrm{~s}$ exposures for two targets and three $30 \mathrm{~s}$ exposures for five targets in a three-position dither pattern that avoided the noisy, lower-left quadrant. We used the narrow camera setting $(0$ ". $0099 / \mathrm{px})$, which gave a single-frame FOV of $10^{\prime \prime} \times 10^{\prime \prime}$.

\subsubsection{SOAR Goodman Spectroscopy}

We took spectra of 24 of the subdwarfs using the Southern Astrophysical Research Telescope (SOAR) and the Goodman Spectrograph (Clemens et al. 2004) on 2014 July 15. We observed 12 targets with companions and 12 single stars from the subdwarf sample as reference. The spectra were taken using a 930 lines/mm grating with $0.42 \AA /$ pixel, a 1 ".07 slit, and exposure times of $480 \mathrm{~s}$.

\section{DATA REDUCTION AND ANALYSIS}

\subsection{Robo-AO Imaging}

\subsubsection{Target Verification}

To verify that each star viewed in the image is the desired subdwarf target, we created Digital Sky Survey cutouts of similar angular size around the target coordinates. Each image was then manually checked to assure no ambiguity in the target star. The vast majority of the targets are in relatively sparse stellar regions. Four of the target stars in crowded fields whose identification was ambiguous were discarded, leaving 344 verified subdwarf targets.

\subsubsection{Point-spread Function (PSF) Subtraction}

To locate close companions, a custom locally optimized PSF subtraction routine (Law et al. 2014) based on the Locally Optimized Combination of Images algorithm (Lafrenière et al. 2007) was applied to centered cutouts of all stars. Successful PSF subtraction requires similar reference images, taken at similar times, with similar instruments, and with reference stars of similar brightnesses. The set of subdwarf observations taken at similar times meet these criteria and were used as references, instead of dedicated reference observations, thus optimizing survey efficiency. This is made possible by the improbability of having a companion in the same position for two different targets.

For each target image and for 20 reference images selected as the closest to the target image in observation time, the region around the star was subdivided into polar sections, five upsampled pixels in radius and $45^{\circ}$ in angle. A locally optimized estimate of the PSF for each section was then generated using a linear combination of the reference PSFs. The algorithm begins with an average over the reference PSFs, then uses a downhill simplex algorithm to optimize the contributions from each reference image to find the best fit to the target image. The optimization is done on several coincident sections simultaneously to minimize the probability of subtracting out a real companion, with only the central region outputted to the final PSF. This also provides smoother transitions between adjacent sections as many of the image pixels were shared in the optimization. 


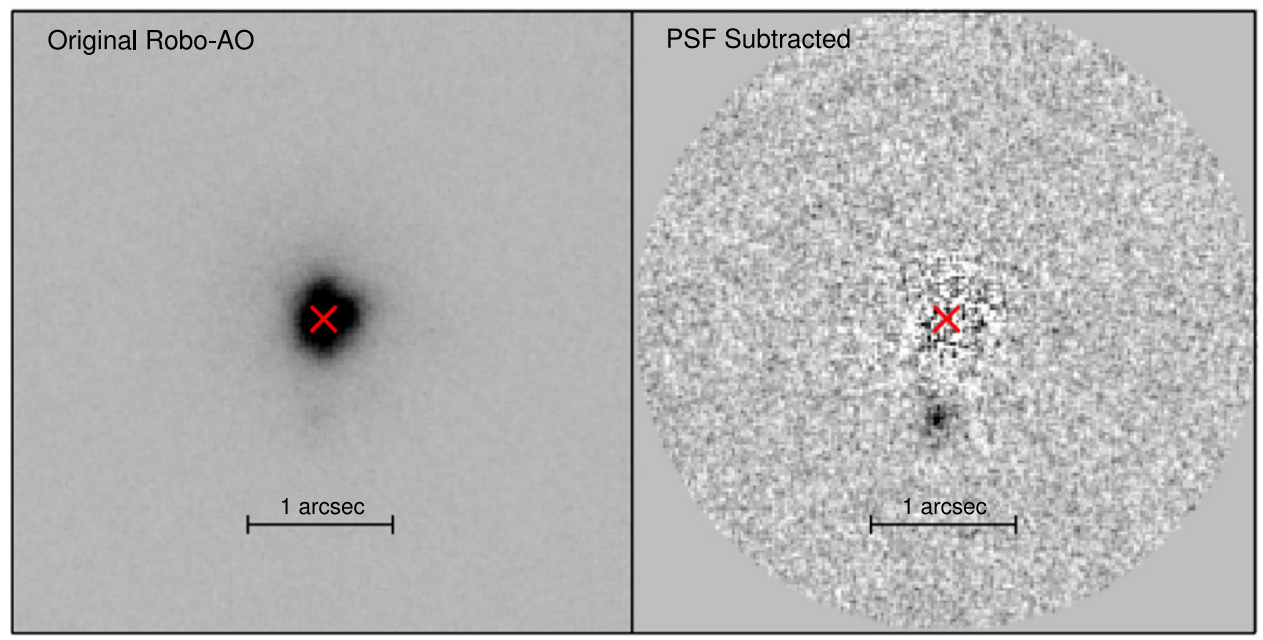

Figure 3. Example of PSF subtraction on NLTT 31240 with companion separation of 0.'74. The red $\times$ marks the position of the primary star's PSF peak. Successful removal of the PSF leaves residuals consistent with photon noise.

After iterating over all sections of the image, the final PSF is an optimal local combination of all the reference PSFs. This final PSF is then subtracted from the original reference image, leaving residuals that are consistent with photon noise. Figure 3 shows an example of the PSF subtraction performance.

We ran the PSF subtraction algorithm on all our targets out to a radius of $2^{\prime \prime}$. We subsequently reran the automated companion detection routine on the subtracted images to find significant $(>5 \sigma)$ close companions, and manually checked the results.

\subsubsection{Automated Companion Detection}

To efficiently find companions in the large data set, we developed a custom search algorithm, based on the method described in Law et al. (2014). The algorithm searches every four-pixel diameter aperture in the image and compares the signal inside the aperture to the average noise level at that radius from the target star. The detected companions were then manually checked, eliminating spurious detections with dissimilar PSFs to the target star and those having characteristics of a cosmic ray hit, such as a single bright pixel or bright streak. The detection significance of found companions is listed in Table 4.

\subsubsection{Imaging Performance Metrics}

The two dominant factors that effect the image performance of the Robo-AO system are seeing and target brightness. To further classify the image performance for each target an automated routine was ran on all images. Described in detail in Law et al. (2014), the code uses two Moffat functions fit to the PSF to separate the widths of the core and halo. We found that the core size was an excellent predictor of the contrast performance, and used it to group targets into three levels (low, medium, and high). Counter-intuitively, the PSF core size decreases as image quality decreases. This is caused by poor signal-to-noise ratio on the shift-and-add image alignment used by the EMCCD detector. The frame alignment subsequently locks onto photon noise spikes, leading to single-pixelsized spikes in the images (Law et al. 2006b, 2009). The images with diffraction limited core size $\left.(\sim 0)^{\prime \prime} 15\right)$ were assigned to the high-performance group, with smaller cores assigned to lower-performance groups. For our target observations, $32 \%$ fall in the low performance group, $43 \%$ in the medium performance group, and $25 \%$ in the high performance group.

Using a companion-detection simulation with a group of representative targets, we determine the angular separation and contrast consistent with a $5 \sigma$ detection. For clarity, the contrast curves of the simulated targets are fitted with functions of the form $a-b /(r-c)$ (where $r$ is the radius from the target star and $a, b$, and $c$ are fitting variables). Contrast curves for the three performance groups are shown in Section 5 in Figure 9 passing reference.

\subsubsection{Contrast Ratios}

For wide companions, the binaries' contrast ratio was determined using aperture photometry on the original images. The aperture size was determined uniquely for each system based on separation and the presence of non-associated background stars.

For close companions, the estimated PSF was used to remove the blended contributions of each star before aperture photometry was performed. The locally optimized PSF subtraction algorithm attempts to remove the flux from companions using other reference PSFs with excess brightness in those areas. For detection purposes, we use many PSF core sizes for optimization, and the algorithm's ability to remove the companion light is reduced. However, the companion is artificially faint as some flux has still been subtracted. To avoid this, the PSF fit was redone excluding a six-pixeldiameter region around the detected companion. The large PSF regions allow the excess light from the primary star to be removed, while not reducing the brightness of the companion.

\subsubsection{Separation and Position Angles}

Separation angles were determined from the raw pixel positions. Uncertainties were found using estimated systematic errors due to blending between components. Typical uncertainty in the position for each star was 1-2 pixels. Position angles were calculated using a distortion solution produced using Robo-AO measurements for a globular cluster. ${ }^{4}$

S. Hildebrandt (2013, private communication). 


\subsection{Previously Detected Binaries}

To further realize our goal of a comprehensive cool subdwarf survey, we included in our statistics previously confirmed binary systems in the literature with separations outside of our FOV. Common proper motion is a useful indicator of wider binary systems. Wide $\left(>30^{\prime \prime}\right)$ common proper motion companions among our target subdwarfs were previously identified in the Revised New Luyten Two-Tenths catalog (rNLTT; Salim \& Gould 2002; Chanamé \& Gould 2004), and a search by López et al. (2012) of the Lepine and Shara Proper MotionNorth catalog (LSPM) (Lépine \& Shara 2005). None of our target stars overlap with the large survey of (Zhang et al. 2013), as our targets are several magnitudes brighter on average.

The target list was also cross-checked against the Ninth Catalogue of Spectroscopic Binary Orbits $\left(S_{B^{9}}\right.$; Pourbaix et al. 2004), a catalog of known spectroscopic binaries available online..$^{5}$ While these systems were included in the total subdwarf binary numbers, the compilatory nature of this catalog leaves some uncertainty in the completeness of the spectroscopic search.

\subsection{Spectroscopy}

To further verify that the targets selected are cool subdwarfs, we took spectra of $7 \%$ of the total survey and $31 \%$ of the candidate companion systems. Past spectroscopic studies of cool subdwarfs at high resolution have proven difficult as, at the low temperatures present, a forest of molecular absorption lines conceals most atomic lines used in spectral analysis. Subdwarfs can be classified spectroscopically using two molecular lines (Gizis 1997). Comparing titanium oxide ( $\mathrm{TiO}$ ) bands to metal hydride bands (typically $\mathrm{CaH}$ in $\mathrm{M}$ subdwarfs), Gizis classified two groups, the intermediate and extreme subdwarfs. As the metallicity decreases, the TiO adsorption also decreases, but the $\mathrm{CaH}$ remains largely unaffected for a given spectral type. This classification system was expanded and revised to include ultra subdwarfs by Lépine et al. (2007), who introduced the new useful parameter $\zeta_{\mathrm{TiO} / \mathrm{CaH}}$.

Spectra were taken for wavelengths 5900-7400 $\AA$, and reduced (dark-subtracted and flat-fielded) using IRAF reduction packages, particularly onedspec.apall to extract the trace of the spectrum and onedspec.dispcor for applying the wavelength calibration. A Fe+Ar arc lamp was recorded for wavelength calibration. All observed target subdwarfs were confirmed to show the spectral characteristics of subdwarf stars described above, specifically the reduced band strength of $7050 \AA$ TiO5. An example of the extracted spectra is given in Figure 4. The full observation list for SOAR is given in Table 2.

With all 24 sampled subdwarfs confirmed, spectroscopy alone gives a $95 \%$ confidence limit that the fractional dwarf contamination is below 0.12 ; the most likely contamination (50th percentile) below 0.03. This does not account, however, for the targets placement on a RPM diagram, which also suggests that the stars are in fact subdwarfs. We expect that the dwarf contamination is thus also lower than the small spectroscopic sample implies. We therefore consider targets not yet observed by SOAR to be probable, although unconfirmed, subdwarfs.

\footnotetext{
http://sb9.astro.ulb.ac.be/
}

\subsection{Candidate Companion Follow-ups}

With either high contrast ratio or small angular separation, seven candidate subdwarf binary systems with low detection significance $(<6 \sigma)$ were selected for follow-up imaging using Keck II. One low-probability candidate companion star was rejected after followups using Keck II, an apparent close $(\rho \simeq 0$ !' 15) binary to NLTT 50869, probably resulting from a cosmic ray on the original Robo-AO image. A wider binary to NLTT 50869, with high detection significance, was not in the image FOV. Outside of the six target stars with low significantce companions, another candidate companion star, NLTT 4817, was observed and had no companion inside the FOV of the Keck II image; however, it had a high significance companion $(>7 \sigma)$ in the Robo-AO FOV. An example of the Keck II images and the Robo-AO images is given in Figure 5. The full Keck II observations are listed in Table 3, with the second to last column indicating the presence of companion and the last column the $H$-band magnitude difference of the companion. Angular separations for the companions are listed in Table 4. Confirmed companions and contrast curve for the Keck images are plotted in Figure 6. The area under the contrast curve was searched for all Keck images and was free of additional companions.

\section{DISCOVERIES}

Of the 344 verified subdwarf targets observed, 43 appear to be in multiple star systems for an apparent binary fraction of $12.5 \pm 1.9 \%$, where the error is based on Poissonian statistics (Burgasser et al. 2003). This count includes six multiple systems first recorded in the NLTT, 13 systems first recorded in the rNLTT, one wide binary found in the LSPM (López et al. 2012), six spectroscopic binaries, and 19 newly discovered multiple systems. We also found five new companions to already recorded binary systems, including two new triple systems, for a total of seven triple star systems, for a triplet fraction of $2.0 \pm 0.8 \%$. One quarter $(26 \%)$ of the companions would only be observable in a high-resolution survey $(<2$ !! 0 separation). The overarching dwarf trend of decreased binary fraction with later spectral types is not apparent for our sample of subdwarfs. This is seen in Figure 7, where the binary fraction of the target stars binned by their $(V-J)$ color is presented. Cutouts of the closest 22 multiple star systems are shown in Figure 8. Measured companion properties are detailed in Table 4.

\subsection{Probability of Association}

The associations of all discovered and previously recorded companions were confirmed using the DSS (Reid et al. 1991), of the POSS-I (Abell 1959). Since all the targets have high proper motions, if not physically associated the systems would have highly apparent shifts in separation and position angle over the past six decades. For the widely separated systems with both stars visible in the DSS, we checked the angular separation in the DSS and our survey to confirm relatively constant separation. For closely separated systems where both stars are merged in the DSS, we looked for a background star at the current position that does not appear in our images.

With the majority of POSS-I archival images taken between between 1949 and 1956 and scanned with plate scale of 1 '/px, we can dissociate stars in the field with proper motion

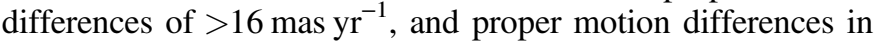




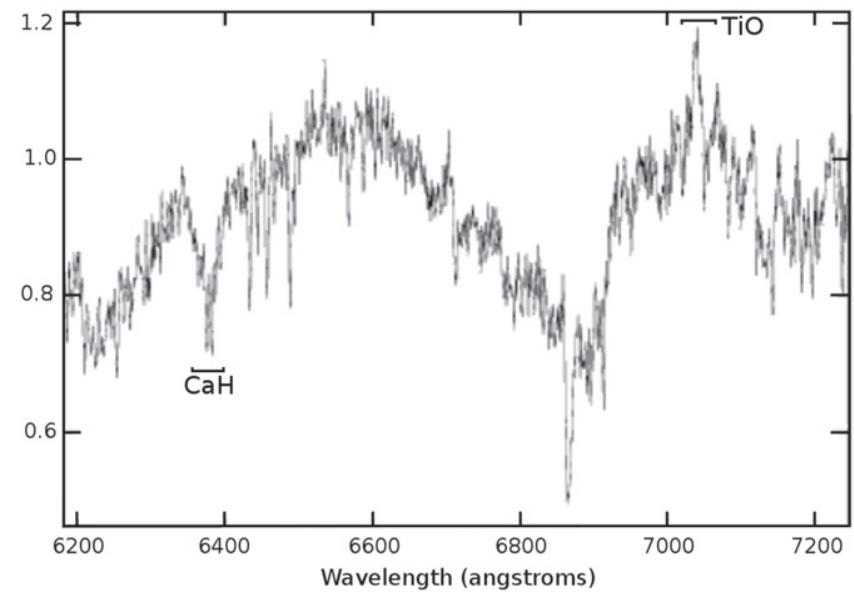

Figure 4. Extracted spectra for NLTT 52532 showing subdwarf characteristics, most apparent the weakness of the $7050 \AA \mathrm{TiO}$ band and strength of the $6380 \AA \mathrm{CaH}$ band. The $y$-axis is given in normalized arbitrary flux units.

Table 2

Full SOAR Spectroscopic Observation List

\begin{tabular}{lccc}
\hline \hline NLTT & $m_{v}$ & ObsID & Companion? \\
\hline 2205 & 14.0 & 2014 Jul 14 14 & Yes \\
7301 & 14.9 & 2014 Jul 14 & Yes \\
7914 & 14.3 & 2014 Jul 14 14 & Yes \\
9597 & 12.0 & 2014 Jul 14 14 & $\ldots$ \\
9898 & 14.2 & 2014 Jul 14 14 & $\ldots$ \\
10022 & 15.8 & 2014 Jul 14 & $\ldots$ \\
10135 & 15.7 & 2014 Jul 14 14 & $\ldots$ \\
33971 & 12.8 & 2014 Jul 14 & $\ldots$ \\
37342 & 14.4 & 2014 Jul 14 & Yes \\
37807 & 12.0 & 2014 Jul 14 & $\ldots$ \\
40022 & 13.9 & 2014 Jul 14 & $\ldots$ \\
40313 & 13.7 & 2014 Jul 14 & $\ldots$ \\
41111 & 13.7 & 2014 Jul 14 & $\ldots$ \\
44039 & 11.5 & 2014 Jul 14 & $\ldots$ \\
44568 & 12.3 & 2014 Jul 14 & $\ldots$ \\
49486 & 16.0 & 2014 Jul 14 & Yes \\
50869 & 15.8 & 2014 Jul 14 & $\ldots$ \\
52377 & 14.5 & 2014 Jul 14 & Yes \\
52532 & 15.5 & 2014 Jul 14 & Yes \\
53255 & 15.0 & 2014 Jul 14 & Yes \\
55603 & 12.1 & 2014 Jul 14 & Yes \\
56818 & 14.0 & 2014 Jul 14 & Yes \\
57038 & 13.9 & 2014 Jul 14 & Yes \\
58812 & 14.9 & 2014 Jul 14 & Yes \\
\hline & & &
\end{tabular}

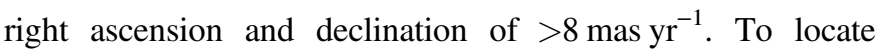
possible fake companions, we use the high-proper motion survey LSPM (Lépine \& Shara 2005), which is estimated over 99\% complete in high galactic latitudes $\left(|b|>15^{\circ}\right)$, where most of our targets lie. Out of approximately 21 million possible associations, we identified 12,451 pairs of stars, one of our subdwarf targets and an LSPM star, which have similar (below our dissociation threshold) proper-motion magnitude and direction. Known associated stars were removed from this sample. With our relatively small FOV and the large sky coverage of the LSPM, the probability of any of these pairs falling within our FOV is a remote $9.1 \times 10^{-5}$.
Table 3

Full Keck-AO Observation List

\begin{tabular}{lcccc}
\hline \hline NLTT & $m_{v}$ & ObsID & Companion? & $\Delta H$ \\
\hline 4817 & 11.4 & 2014 Aug 17 & $\ldots$ & $\ldots$ \\
7914 & 14.3 & 2014 Aug 17 & Yes & 3.83 \\
50869 & 15.8 & 2014 Aug 17 & $\ldots$ & $\ldots$ \\
52377 & 14.5 & 2014 Aug 17 & Yes & 2.64 \\
52532 & 15.5 & 2014 Aug 17 & Yes & 0.53 \\
53255 & 15.0 & 2014 Aug 17 & Yes & 0.64 \\
56818 & 14.0 & 2014 Aug 17 & Yes & 0.69 \\
\hline
\end{tabular}

In addition, since our stars appear in relatively sparse stellar regions in the sky, well outside the Galactic disk, the probability of a background star appearing in a close radius to our observed star is low. Using the total number of known non-associated stars in our images, than at $95 \%$ confidence 7 of the 10 stars found within 2".5 of any of our background stars are associated, with 9 of 10 being the most likely number of associated stars. The small number of probable unassociated background stars in our fields and the DSS proper-motion confirmations suggest a high-likelihood for true association for all of our companion stars.

\subsection{Photometric Parallaxes}

Very few subdwarfs in our sample have accurate parallax measurements. Only 43 of the targets have published parallaxes, most with significant measurement errors. To estimate the distances to our subdwarf targets, we employed an expression for $M_{R}=f(R-I)$ estimated by Siegel et al. (2002) using a color-magnitude diagram and the photometric measurements by Marshall (2007).

The polynomial fit found by Siegel for subdwarfs with measured parallaxes and an estimated mean $[\mathrm{Fe} / \mathrm{H}]$ of -1.2 , and with the Lutz \& Kelker (1973) correction, is

$$
M_{R}=2.03+10 \times(R-I)-2.21 \times(R-I)^{2} .
$$

The color-absolute magnitude relation has an uncertainty of $\sim 0.3$ mag. In all cases, the published parallax errors are much larger than photometric errors of $<0.03$ mag. The estimated distances for the primary stars in the subdwarf multiple systems are listed in Table 4.

\section{DISCUSSION}

\subsection{Comparison to Main-sequence Dwarfs}

With comparable sample size and spectrum types, the cool dwarf survey of Janson et al. (2012) is a useful metal-rich analog to this work. The study used the Lucky Imaging technique on a sample of 761 stars, sensitive to companion separations of $0 .{ }^{\prime \prime} 08-6{ }^{\prime \prime} 0$. The most striking disparity between the two samples is the lack of low-contrast $\left(\Delta m_{i} \leqslant 2\right)$, close $\left(\rho \leqslant 1^{\prime \prime}\right)$ companions to the subdwarf stars, a regime heavily populated by solar-metallicity dwarf companions. This is clearly seen in a plot of the companion's magnitude difference versus angular separation for the two populations, as in Figure 9.

The dissimilarity between contrast ratios between dwarfs and subdwarfs is further illustrated in Figure 10. A two sample Kolmogrov-Smirnov test rejects the null hypothesis that the two populations are similar at a confidence of $\sim 2.8 \sigma$. 

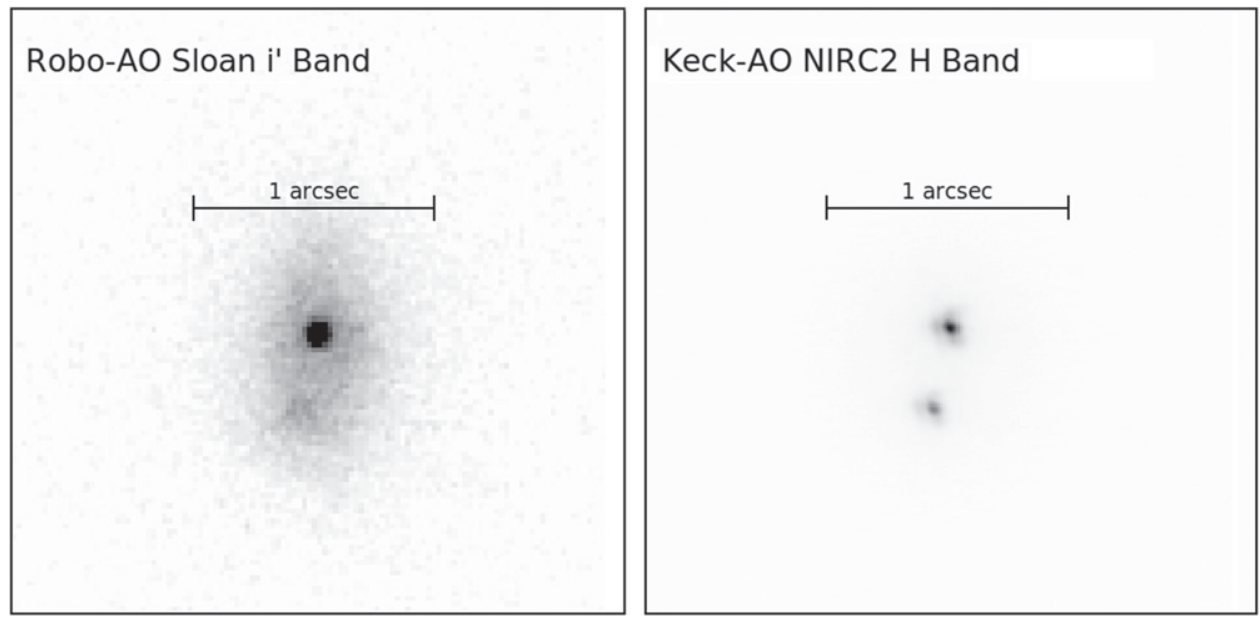

Figure 5. Keck-AO iamge confirming the Robo-AO companion to NLTT 52532. The exposure times are $120 \mathrm{~s}$ for the Robo-AO image and $90 \mathrm{~s}$ for the Keck image.

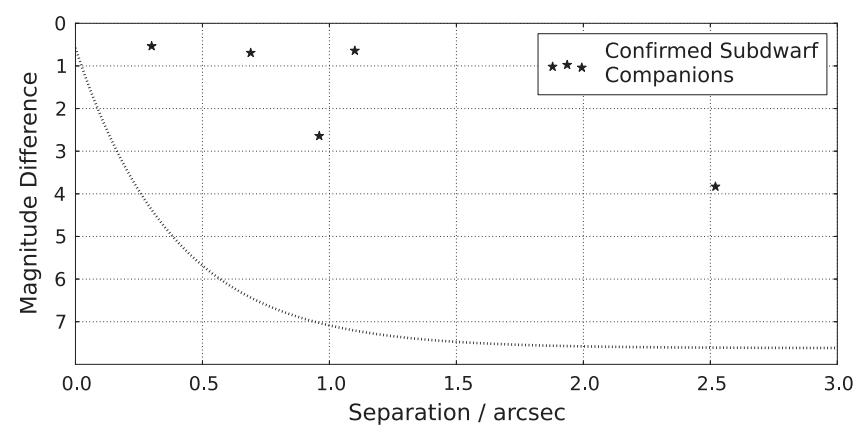

Figure 6. Plot of angular separation and $H$-band magnitude contrast for Keck confirmed Subdwarf companions. The detectable magnitude ratios for the Keck images is plotted, as described in Section 3.1.4.

The lack of close subdwarf companions has been noted previously by Jao et al. (2009) and by Abt (2008), but with significantly smaller samples. A direct comparison of orbital separations is biased by the distance variation in the two samples. With their rarity in the solar neighborhood, the subdwarf sample is overall approximately a factor of 4 further distant than the dwarf sample. If the populations were similar, this would result in a relative abundance of tight dwarf binaries, while the $6^{\prime \prime}$ limit of the Janson et al. survey reduces the number of observed wide dwarf binaries. Attempts to pick out similar systems by relative distance or by orbital separation from the two surveys results in a small statistical sample. Nonetheless, the relative lack of close stars in the subdwarfs sample, as illustrated in Figure 11, and confirmed at highconfidence in our survey, warrants further investigation.

\subsection{Binarity and Metallicity}

The binary fraction we have found further confirms what has been suspected by past studies: that the binary fraction of subdwarfs is substantially lower than their dwarf cousins. The largest survey of cool subdwarfs, although limited by the low angular resolution of the SDSS, Zhang et al. (2013), find a multiplicity for type late $\mathrm{K}$ and $\mathrm{M}$-subdwarfs of $2.41 \%$, with an estimated lower bound of $10 \%$ when adjusting for survey incompleteness. This estimate and our work leave subdwarfs

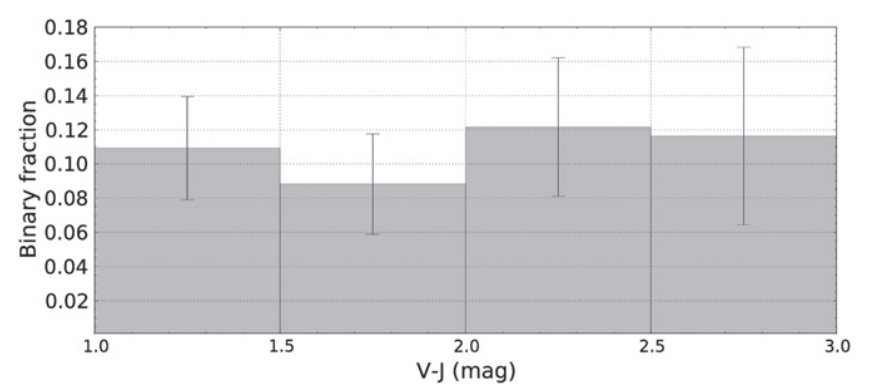

Figure 7. Binary fraction of the target subdwarfs binned by their $(V-J)$ color. The error bars were derived using binomial statistics.

multiplicity rates approximately a factor of 2-4 lower than solar-metallicity stars of the same spectral types.

Historically, it has been a widely held view that metal-poor stars possess fewer stellar companions (Batten 1973; Latham 2004). A deficiency of eclipsing binaries was found in globular clusters by Kopal (1959), while Jaschek \& Jaschek (1959) discovered a deficiency of spectroscopic binaries in a sample of high-velocity dwarfs. Abt \& Willmarth (1987) used higher resolution CCD spectra to conclude that the frequency of spectroscopic binaries in high-velocity stars was half of metal-rich stars. Recently, however, this view has come under attack. Carney et al. (1994) used radial velocity measurements of 1464 stars, along with metallicity data (Carney et al. 1987), and found the difference in binary frequency of metal-rich and metal-poor stars to not be significant. Likewise, Grether \& Lineweaver (2007) found a $\sim 2 \sigma$ anti-correlation between metallicity and companion stars.

In recent years, the relationship between planetary systems and metallicity has also been explored. Fischer \& Valenti (2005) found a positive correlation between planetary systems and the metallicity of the host star. This correlation has been reinforced to $\sim 4 \sigma$ by Grether \& Lineweaver (2007). Recently, Wang et al. (2014) found that planets in multiple-star systems occur $4.5 \pm 3.2,2.6 \pm 1.0$, and $1.7 \pm 0.5$ times less frequently when the companion star is separated by 10, 100, and $1000 \mathrm{AU}$, respectively.

The solution may lie in the differences between halo and thick disk stars. Latham et al. (2002) found no obvious difference between the binary fraction of the two populations; 


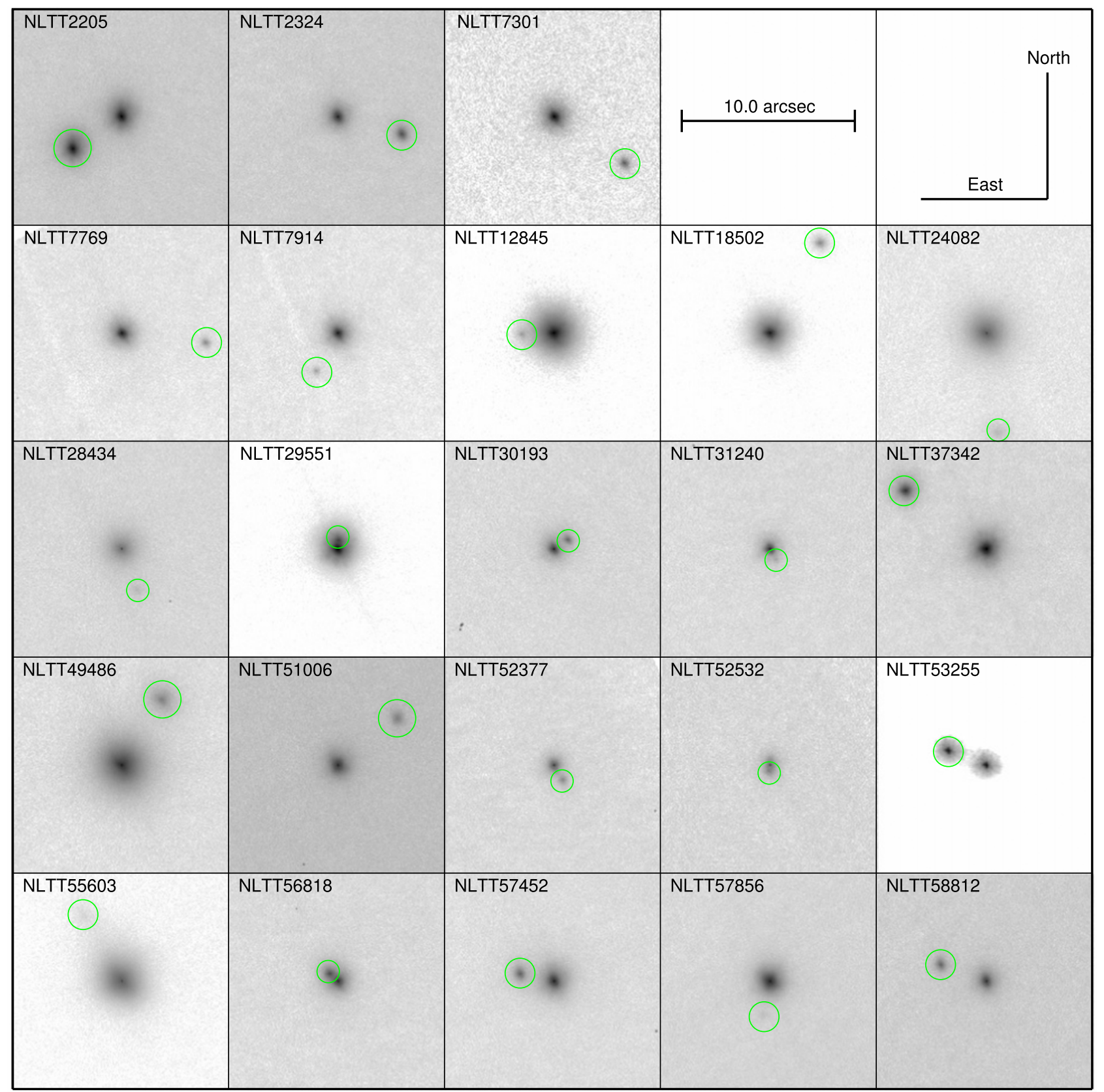

Figure 8. Color inverted, log-scale cutouts of the 23 multiple star systems with separations $<6^{\prime \prime}$ resolved with Robo-AO. The angular scale and orientation is similar for each cutout. The companions to NLTT 7914, 52377, 52532, and 56818 were confirmed with Keck II.

however Chiba \& Beers (2000) found a 55\% multiplicity rate for thick disk stars and $12 \%$ for halo stars. Grether \& Lineweaver also find that the thick disk shows a $\sim 4$ times higher binary fraction than halo stars, further hypothesizing that the mixing of the populations is the explanation for the perceived anti-correlation of metallicity and binarity. Similarly, Bovy et al. (2012) use spectroscopic data to chart the disk thickness, finding, instead of a bi-modal separation of the thin and thick disks, a smooth, continuous distribution. This suggests the absence of a distinct thick disk in the Milky Way.

The large difference between the M-subdwarfs and thickdisk M-dwarfs, apparent in our work in this paper and Janson et al. (2012), seems to imply the two populations formed under different initial conditions. Star formation in less dense regions appears to lower binary rates. Köhler et al. (2006) found a factor 3-5 difference in binary fraction between the low-density Taurus star-forming region and the dense Orion cluster. It is also possible that, as forming earlier than solar-abundance stars, the metal-poor subdwarfs could have suffered more disruptive encounters with other stars and the Galactic tide (Kaib et al. 2013). These disturbances could separate companions with separations larger than a few AU, with the tighter, more highly bound systems being less affected (Sterzik \& Durisen 1998; Abt 2008), a theory derived from $N$-body simulations (Aarseth \& Hills 1972; Kroupa 1995; Jiang \& Tremaine 2010). This, however, is contrary to our tentative result of a lack of close subdwarf companions, and the similar observations of Jao 
Table 4

Multiple Subdwarf Systems Resolved Using Robo-AO and Previously Detected Systems

\begin{tabular}{|c|c|c|c|c|c|c|c|c|c|c|}
\hline NLTT & $\begin{array}{c}\text { Comp } \\
\text { (NLTT) }\end{array}$ & $\begin{array}{c}m_{v}{ }^{\mathrm{a}} \\
(\mathrm{mag})\end{array}$ & ObsID & $\begin{array}{c}\text { Signif. } \\
(\sigma)\end{array}$ & $\begin{array}{c}\Delta \mathrm{i}^{\prime} \\
(\mathrm{mag})\end{array}$ & $\begin{array}{c}\rho \\
\left({ }^{\prime \prime}\right)\end{array}$ & $\begin{array}{c}\rho \\
(\mathrm{AU})\end{array}$ & $\begin{array}{l}\text { P.A. } \\
\text { (deg.) }\end{array}$ & $\begin{array}{l}\text { Dist } \\
(\mathrm{pc})\end{array}$ & $\overline{\text { Prev Det? }}$ \\
\hline $2045 \mathrm{AB}$ & $\ldots$ & 13.5 & 2013 Aug 15 & $\ldots$ & $\ldots$ & $\ldots$ & $\ldots$ & $\ldots$ & $183.3 \pm 21.0$ & SB 9 \\
\hline $2205 \mathrm{AB}$ & 2206 & 13.9 & 2013 Aug 15 & 52 & 0.18 & 3.37 & $475.5 \pm 54.3$ & $123 \pm 2$ & $140.9 \pm 16.1$ & L79 \\
\hline $2324 \mathrm{AB}$ & 2325 & 15.7 & 2013 Aug 16 & 19 & 1.16 & 3.84 & $138.8 \pm 15.9$ & $254 \pm 2$ & $36.1 \pm 4.1$ & L79 \\
\hline $2324 \mathrm{AC}$ & $\ldots$ & 15.7 & 2013 Aug 16 & 16 & 4.14 & 23.48 & $847.8 \pm 96.2$ & $159 \pm 2$ & $36.1 \pm 4.1$ & $\ldots$ \\
\hline $4817 \mathrm{AB}$ & 4814 & 11.4 & 2012 Sep 3 & 65 & 4.30 & 24.59 & $3615 \pm 413$ & $218 \pm 2$ & $147 \pm 16.8$ & S02 \\
\hline $7301 \mathrm{AB}$ & 7300 & 14.9 & 2012 Sep 3 & 30 & 2.48 & 4.87 & $105.7 \pm 12.1$ & $57 \pm 2$ & $21.7 \pm 2.5$ & $\mathrm{~S} 02$ \\
\hline $7769 \mathrm{AB}$ & $\cdots$ & 14.0 & 2012 Sep 3 & 8.2 & 3.34 & 4.84 & $1106 \pm 126$ & $121 \pm 2$ & $228.6 \pm 26.2$ & $\ldots$ \\
\hline $7914 \mathrm{AB}$ & $\ldots$ & 14.3 & 2012 Sep 3 & $39^{\mathrm{b}}$ & 3.76 & $2.53^{\mathrm{b}}$ & $424.4 \pm 48.5$ & $150 \pm 2$ & $167.6 \pm 19.2$ & $\ldots$ \\
\hline $10536 \mathrm{AB}$ & 10548 & 11.2 & 2013 Aug 15 & $\ldots$ & $\cdots$ & 185.7 & $30633 \pm 3501$ & 85.5 & $164.9 \pm 18.9$ & S02 \\
\hline $11015 \mathrm{AB}$ & 11016 & 16.3 & 2013 Aug 16 & 42 & 0.94 & 9.24 & $1399 \pm 160$ & $57 \pm 2$ & $151.3 \pm 17.3$ & S02 \\
\hline $12845 \mathrm{AB}$ & $\ldots$ & 10.6 & 2012 Oct 3 & 49 & 4.71 & 1.85 & $149.4 \pm 17.1$ & $92 \pm 2$ & $80.6 \pm 9.2$ & $\ldots$ \\
\hline $15973 \mathrm{AB}$ & 15974 & 9.3 & 2012 Oct 7 & 22 & 3.47 & 6.88 & $303.1 \pm 34.6$ & $227 \pm 2$ & $44 \pm 5.0$ & S02 \\
\hline $15973 \mathrm{AC}$ & $\ldots$ & 9.3 & 2012 Oct 7 & 7.2 & 5.02 & 8.23 & $362.2 \pm 41.1$ & $217 \pm 2$ & $44 \pm 5.0$ & $\ldots$ \\
\hline $17485 \mathrm{AB}$ & $\ldots$ & 11.9 & 2012 Oct 10 & $\ldots$ & $\ldots$ & $\ldots$ & $\ldots$ & $\ldots$ & $191.3 \pm 21.9$ & SB 9 \\
\hline $18502 \mathrm{AB}$ & $\cdots$ & 12.2 & 2013 Jan 19 & 25 & 3.18 & 5.95 & $1262 \pm 144$ & $331 \pm 2$ & $212.1 \pm 24.3$ & $\ldots$ \\
\hline $18798 \mathrm{AB}$ & 18799 & 14.5 & 2013 Jan 19 & 48 & 3.12 & 12.82 & $2270 \pm 259$ & $172 \pm 2$ & $177 \pm 20.2$ & S02 \\
\hline $19210 \mathrm{AB}$ & 19207 & 11.2 & 2013 Jan 20 & $\ldots$ & $\ldots$ & 102.5 & $18468 \pm 2110$ & 285.4 & $180.2 \pm 20.6$ & S02,SB 9 \\
\hline $20691 \mathrm{AB}$ & $\cdots$ & 9.6 & 2013 Jan 19 & 12 & 5.47 & 1.52 & $107.3 \pm 12.3$ & $93 \pm 2$ & $70.6 \pm 8.1$ & SB 9 \\
\hline $21370 \mathrm{AB}$ & $\ldots$ & 13.7 & 2013 Jan 19 & 71 & 2.46 & 19.83 & $6603 \pm 755$ & $322 \pm 2$ & $332.9 \pm 38.1$ & SB 9 \\
\hline $24082 \mathrm{AB}$ & $\cdots$ & 13.1 & 2013 Jan 19 & 4.8 & 4.46 & 5.81 & $1683 \pm 192$ & $187 \pm 2$ & $289.7 \pm 33.1$ & $\ldots$ \\
\hline $24082 \mathrm{AC}$ & $\ldots$ & 13.1 & 2013 Jan 19 & 3.8 & 4.17 & 12.00 & $3476 \pm 397$ & $267 \pm 2$ & $289.7 \pm 33.1$ & $\ldots$ \\
\hline $25234 \mathrm{AB}$ & 25233 & 13.2 & 2013 Jan 18 & 65 & 3.05 & 8.29 & $1175 \pm 134$ & $287 \pm 2$ & $141.7 \pm 16.2$ & S02 \\
\hline $28434 \mathrm{AB}$ & $\ldots$ & 14.9 & 2013 Jan 17 & 2.2 & 2.46 & 2.54 & $652.9 \pm 74.6$ & $202 \pm 2$ & $256.7 \pm 29.3$ & $\ldots$ \\
\hline $29551 \mathrm{AB}$ & $\cdots$ & 11.5 & 2012 Sep 3 & 8.9 & 3.29 & 0.51 & $104.6 \pm 12.0$ & $355 \pm 2$ & $206.5 \pm 23.6$ & $\cdots$ \\
\hline $29594 \mathrm{AB}$ & $\ldots$ & 13.2 & 2013 Apr 22 & $\ldots$ & $\ldots$ & 38.10 & $12834 \pm 1466$ & 269 & $336.8 \pm 38.5$ & L12 \\
\hline 30193AB & $\ldots$ & 14.6 & 2013 Apr 21 & 12 & 1.99 & 0.95 & $304.8 \pm 34.8$ & $304 \pm 2$ & $321.5 \pm 36.7$ & $\ldots$ \\
\hline $30838 \mathrm{AB}$ & 30837 & 12.5 & 2013 Apr 22 & 11 & 5.69 & 16.25 & $4436 \pm 507$ & $25 \pm 2$ & $273 \pm 31.2$ & S02 \\
\hline $31240 \mathrm{AB}$ & $\ldots$ & 15.0 & 2013 Apr 21 & 13 & 3.86 & 10.32 & $3491 \pm 399$ & $157 \pm 2$ & $338.3 \pm 38.7$ & $\ldots$ \\
\hline $31240 \mathrm{AC}$ & $\ldots$ & 15.0 & 2013 Apr 21 & 5.1 & 4.16 & 0.74 & $251.2 \pm 28.7$ & $210 \pm 2$ & $338.3 \pm 38.7$ & $\ldots$ \\
\hline $34051 \mathrm{AB}$ & $\ldots$ & 13.5 & 2013 Jan 19 & $\ldots$ & $\ldots$ & $\ldots$ & $\ldots$ & $\ldots$ & $242.3 \pm 27.7$ & SB 9 \\
\hline $37342 \mathrm{AB}$ & 37341 & 14.4 & 2013 Apr 22 & 49 & 1.37 & 5.75 & $123.4 \pm 14.1$ & $54 \pm 2$ & $21.4 \pm 2.5$ & S02 \\
\hline $45616 \mathrm{AB}$ & $\ldots$ & 11.9 & 2012 Sep 3 & 125 & 2.59 & 28.31 & $4696 \pm 536.8$ & $113 \pm 2$ & $165.9 \pm 19.0$ & SB 9 \\
\hline 49486AB & 49487 & 15.9 & 2012 Oct 4 & 9.3 & 1.48 & 4.51 & $390.3 \pm 44.6$ & $148 \pm 2$ & $86.4 \pm 9.9$ & $\mathrm{~S} 02$ \\
\hline 49819AB & 49821 & 14.0 & 2013 Aug 19 & 340 & 1.12 & 25.28 & $10263 \pm 1173$ & $84 \pm 2$ & $406 \pm 46.4$ & $\mathrm{~S} 02$ \\
\hline $50759 \mathrm{AB}$ & $\ldots$ & 15.9 & 2012 Sep 13 & 24 & 2.02 & 13.33 & $3544 \pm 405$ & $26 \pm 2$ & $265.8 \pm 30.4$ & $\ldots$ \\
\hline 50759AC & 50751 & 15.9 & 2012 Sep 13 & $\cdots$ & $\cdots$ & 297.7 & $79156 \pm 9046$ & 267.7 & $265.8 \pm 30.4$ & S02 \\
\hline $50869 \mathrm{AB}$ & $\ldots$ & 15.8 & 2013 Aug 8 & 7.4 & 3.15 & 8.17 & $1707 \pm 195$ & $19 \pm 2$ & $209.0 \pm 24.0$ & $\ldots$ \\
\hline $51006 \mathrm{AB}$ & $\ldots$ & 14.0 & 2013 Aug 8 & 5.2 & 2.23 & 4.35 & $961.8 \pm 109.9$ & $76 \pm 2$ & $221.1 \pm 25.3$ & $\ldots$ \\
\hline $52377 \mathrm{AB}$ & $\ldots$ & 14.5 & 2012 Sep 4 & $568^{\mathrm{b}}$ & 2.35 & $0.92^{\mathrm{b}}$ & $561.3 \pm 64.2$ & $211 \pm 2$ & $585.3 \pm 66.9$ & $\ldots$ \\
\hline $52532 \mathrm{AB}$ & $\ldots$ & 15.5 & 2012 Sep 4 & $14^{\mathrm{b}}$ & 2.60 & $0.30^{\mathrm{b}}$ & $52.82 \pm 6.0$ & $168 \pm 2$ & $175 \pm 20.0$ & $\ldots$ \\
\hline $52532 \mathrm{AC}$ & 52538 & 15.5 & 2012 Sep 4 & $\ldots$ & 3.35 & 37.14 & $6536 \pm 780$ & $\ldots$ & $176 \pm 21.0$ & L79 \\
\hline $53255 \mathrm{AB}$ & $\ldots$ & 15.0 & 2013 Aug 16 & $58^{\mathrm{b}}$ & 0.75 & $1.07^{\mathrm{b}}$ & $123.9 \pm 14.2$ & $68 \pm 2$ & $112.7 \pm 12.9$ & $\ldots$ \\
\hline $53255 \mathrm{AC}$ & 53254 & 15.0 & 2013 Aug 16 & $\cdots$ & $\cdots$ & 53.8 & $6063 \pm 694$ & $\ldots$ & $112.7 \pm 12.9$ & L79 \\
\hline $53274 \mathrm{AB}$ & $\ldots$ & 11.8 & 2013 Aug 17 & 5.0 & 5.75 & 6.17 & $555.9 \pm 63.5$ & $153 \pm 2$ & $90.1 \pm 10.3$ & $\ldots$ \\
\hline $55603 \mathrm{AB}$ & $\ldots$ & 12.1 & 2013 Aug 18 & 2.6 & 3.54 & 4.45 & $886.9 \pm 101.4$ & $29 \pm 2$ & $199.2 \pm 22.8$ & $\ldots$ \\
\hline $56818 \mathrm{AB}$ & $\ldots$ & 14.0 & 2012 Sep 3 & $60^{\mathrm{b}}$ & 2.04 & $0.63^{\mathrm{b}}$ & $169.8 \pm 19.4$ & $44 \pm 2$ & $246.2 \pm 28.1$ & $\ldots$ \\
\hline $57038 \mathrm{AB}$ & $\ldots$ & 13.9 & 2013 Aug 16 & 210 & 0.19 & 8.14 & $2508 \pm 286.7$ & $335 \pm 2$ & $308.3 \pm 35.2$ & $\ldots$ \\
\hline $57452 \mathrm{AB}$ & $\ldots$ & 13.6 & 2013 Aug 16 & 14 & 1.91 & 1.98 & $474.5 \pm 54.2$ & $77 \pm 2$ & $234.9 \pm 26.9$ & $\ldots$ \\
\hline $57856 \mathrm{AB}$ & $\ldots$ & 13.2 & 2013 Aug 17 & 2.0 & 5.08 & 2.00 & $585.3 \pm 66.9$ & $169 \pm 2$ & $289.7 \pm 33.1$ & $\ldots$ \\
\hline $58812 \mathrm{AB}$ & 58813 & 15.0 & 2013 Aug 16 & 10 & 1.40 & 2.81 & $743.6 \pm 85.0$ & $69 \pm 2$ & $264.4 \pm 30.2$ & $\ldots$ \\
\hline
\end{tabular}

Notes. References for previous detections are denoted using the following codes: Pourbaix et al. (2004) (SB 9), Luyten (1979) (L79), Saliom \& Gould (2002) (S02), López et al. (2012) (L12).

a (Marshall 2007).

${ }^{\mathrm{b}}$ From Keck follow-up, described in Section 3.4.

et al. (2009) and Abt (2008) that close subdwarf binaries are rare. This implies that metal-poor subdwarfs had shorter lifetimes in clusters than their younger, metal-rich cousins, either being ejected or formed in a disrupted cluster.

Another possible explanation is that a large number of lowmetallicity stars in the Milky Way could have resulted from past mergers with satellite galaxies. Simulations from Abadi et al. (2006) predict that the early Galaxy underwent a period of active merging. From these mergers, the Galaxy would inherit large numbers of metal-poor stars. Meza et al. (2005) observe a group of metal-poor stars with angular momenta similar to the cluster $\omega$ Cen, long theorized to be the core of a 


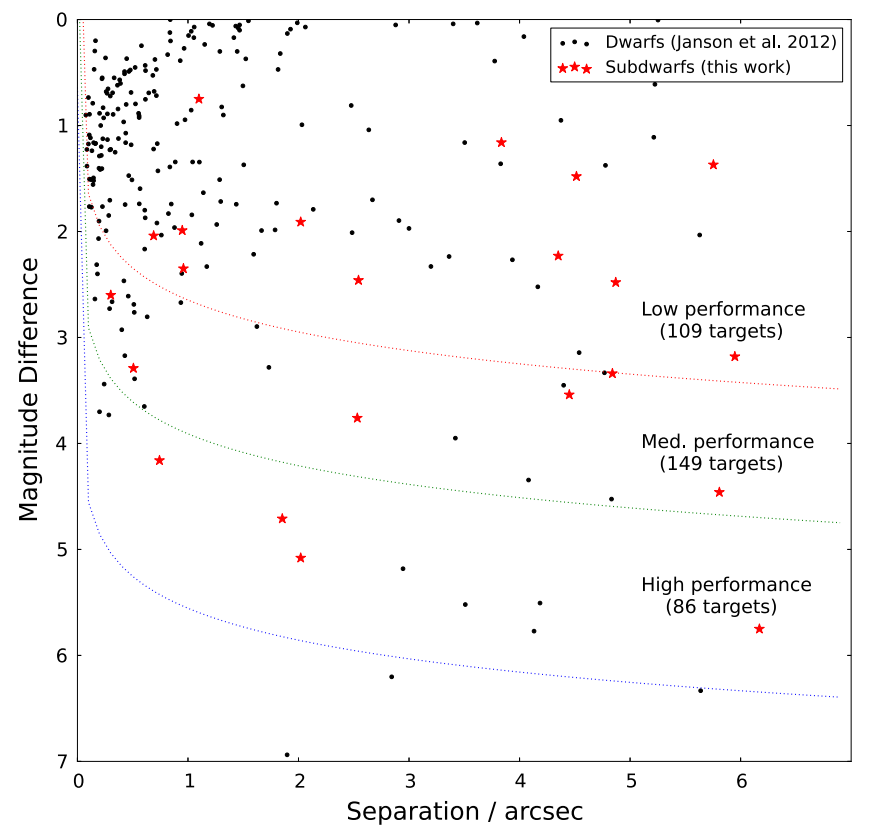

Figure 9. Comparison of the separation and the magnitude difference in the iband between our subdwarf companions $\left(<6^{\prime \prime}\right)$ and the dwarf companions found by Janson et al. (2012). The detectable magnitude ratios for our image performance groups are also plotted, with the number of observed subdwarf targets in each image performance group, as described in Section 3.1.4.

dwarf galaxy that merged with the Milky Way. The environment of these foreign galaxies is unknown, so star formation could be quite different than our own Galaxy. It is also possible that during the merger multiple close stellar encounters and perturbations could alter their primordial binary properties.

\section{CONCLUSIONS}

In the largest high-resolution binary survey of cool subdwarfs, we observed 344 stars with the Robo-AO robotic laser adaptive optics system, sensitive to companions at $\rho \geqslant 0$." 15 and $\Delta m_{i} \leqslant 6$. Of those targets, we observed 16 new multiple systems and five new companions to already known binary systems. When including previously recorded multiple systems, this implies a multiplicity rate for cool subdwarfs of 12.5 $\pm 1.9 \%$ and a triplet fraction of $2.0 \pm 0.8 \%$. This is significantly lower than the observed cool subdwarf binarity of $26 \pm 6 \%$ by Jao et al. (2009) and in agreement with the completeness adjusted estimate of $>10 \%$ of Zhang et al. (2013). When comparing our results to similar surveys of nonsubdwarf binarity, we note a $\sim 2.8 \sigma$ difference in relative magnitude differences between companions. An apparent lack of close binaries is noted, as has been previously observed in the literature. The high efficiency of Robo-AO makes large, high-angular resolution surveys practical and will in the future continue to put tighter constraints on the properties of stellar populations.

We would like to acknowledge the anonymous referee for careful analysis of our paper and the comments that have improved it. The Robo-AO system is supported by collaborating partner institutions, the California Institute of Technology and the Inter-University Centre for Astronomy and Astrophysics, and by the National Science Foundation under Grant Nos. AST-0906060, AST-0960343, and AST-1207891, by the Mount Cuba Astronomical Foundation, and by a gift from

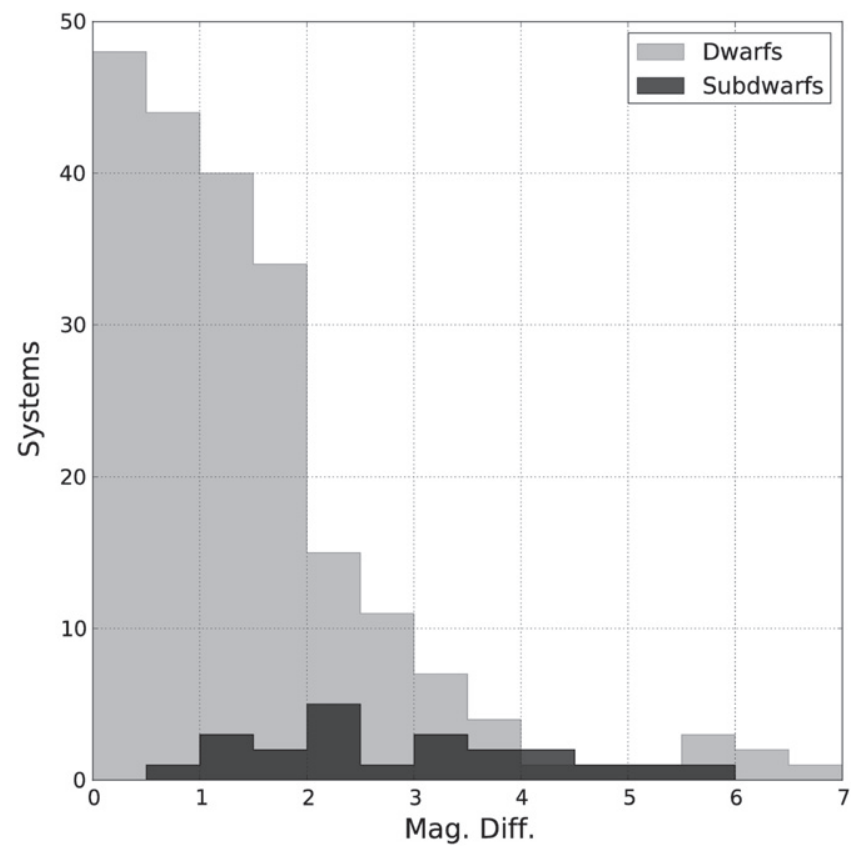

Figure 10. Histogram of the magnitude difference in the $i$-band between all our subdwarf companions and the dwarf companions found by Janson et al. (2012).

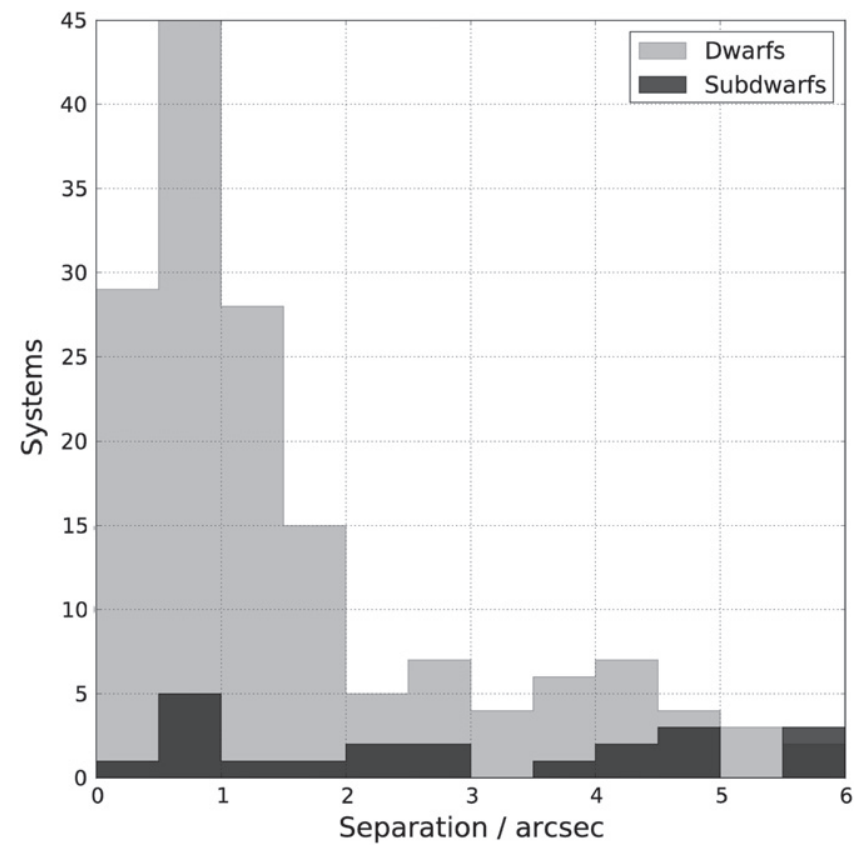

Figure 11. Histogram of the angular separations of our subdwarf companions and the dwarf companions found by Janson et al. (2012). Only systems resolvable in both surveys were plotted $\left(0 .{ }^{\prime \prime} 15<\rho<6 . " 0\right)$.

Samuel Oschin. We are grateful to the Palomar Observatory staff for their ongoing support of Robo-AO on the 60 inch telescope, particularly S. Kunsman, M. Doyle, J. Henning, R. Walters, G. Van Idsinga, B. Baker, K. Dunscombe, and D. Roderick. The SOAR telescope is operated by the Association of Universities for Research in Astronomy, Inc., under a cooperative agreement between the $\mathrm{CNPq}$, Brazil, the National Observatory for Optical Astronomy (NOAO), the University of North Carolina, and Michigan State University, USA. We also 
Table A1

Full Robo-AO Observation List

\begin{tabular}{|c|c|c|c|c|}
\hline NLTT & $m_{v}$ & ObsID & Obs. Qual & Companion? \\
\hline 69 & 15.2 & 2012 Oct 10 & Low & $\ldots$ \\
\hline 193 & 15.5 & 2013 Aug 15 & Medium & $\cdots$ \\
\hline 341 & 12.1 & 2012 Oct 10 & High & $\cdots$ \\
\hline 361 & 15.4 & 2013 Aug 17 & Low & $\cdots$ \\
\hline 496 & 15.8 & 2012 Sep 04 & Medium & $\cdots$ \\
\hline 660 & 15.7 & 2012 Sep 03 & Low & $\cdots$ \\
\hline 812 & 12.8 & 2012 Sep 03 & High & $\cdots$ \\
\hline 933 & 15.5 & 2013 Aug 16 & Low & $\cdots$ \\
\hline 1020 & 15.3 & 2013 Aug 15 & Medium & $\cdots$ \\
\hline 1059 & 13.8 & 2012 Sep 04 & Medium & $\cdots$ \\
\hline 1231 & 11.9 & 2013 Aug 16 & High & $\cdots$ \\
\hline 1509 & 15.8 & 2013 Aug 16 & Low & $\cdots$ \\
\hline 1575 & 16.2 & 2012 Sep 03 & Low & $\cdots$ \\
\hline 1635 & 13.2 & 2012 Sep 03 & High & $\cdots$ \\
\hline 1684 & 15.1 & 2012 Sep 13 & Low & $\cdots$ \\
\hline 1815 & 15.5 & 2012 Sep 04 & Low & $\cdots$ \\
\hline 1870 & 13.9 & 2012 Sep 03 & Medium & $\cdots$ \\
\hline 2045 & 13.5 & 2013 Aug 15 & Medium & Yes \\
\hline 2107 & 15.5 & 2012 Sep 04 & Low & $\cdots$ \\
\hline 2205 & 14.0 & 2013 Aug 15 & Medium & Yes \\
\hline 2324 & 15.7 & 2013 Aug 16 & Medium & Yes \\
\hline 2868 & 13.5 & 2013 Aug 16 & Medium & $\cdots$ \\
\hline 2953 & 15.9 & 2012 Sep 04 & Low & $\cdots$ \\
\hline 2966 & 15.6 & 2012 Sep 04 & Medium & $\cdots$ \\
\hline 3035 & 15.9 & 2012 Sep 04 & Low & $\cdots$ \\
\hline 3965 & 16.1 & 2013 Aug 16 & Medium & $\cdots$ \\
\hline 4245 & 15.6 & 2013 Aug 15 & Low & $\cdots$ \\
\hline 4447 & 15.9 & 2012 Sep 03 & Low & $\cdots$ \\
\hline 4817 & 11.4 & 2012 Sep 03 & High & Yes \\
\hline 4838 & 15.4 & 2012 Sep 03 & Low & $\cdots$ \\
\hline 5022 & 13.9 & 2012 Sep 03 & Medium & $\cdots$ \\
\hline 5192 & 14.3 & 2012 Sep 03 & Medium & $\cdots$ \\
\hline 5289 & 15.6 & 2012 Sep 03 & Low & $\cdots$ \\
\hline 6519 & 14.8 & 2012 Sep 03 & Medium & $\cdots$ \\
\hline 6582 & 15.7 & 2013 Aug 17 & Low & $\cdots$ \\
\hline 6614 & 15.7 & 2012 Sep 03 & Medium & $\cdots$ \\
\hline 6816 & 16.1 & 2013 Aug 15 & Low & $\cdots$ \\
\hline 6856 & 16.1 & 2012 Sep 03 & Low & $\cdots$ \\
\hline 6863 & 15.3 & 2013 Aug 17 & Low & $\cdots$ \\
\hline 7078 & 14.4 & 2012 Sep 03 & Medium & $\cdots$ \\
\hline 7207 & 14.5 & 2013 Aug 15 & Medium & $\cdots$ \\
\hline 7299 & 11.5 & 2013 Aug 16 & High & $\cdots$ \\
\hline 7301 & 14.9 & 2012 Sep 03 & High & Yes \\
\hline 7415 & 9.1 & 2012 Sep 03 & High & $\cdots$ \\
\hline 7417 & 11.6 & 2013 Aug 15 & High & $\cdots$ \\
\hline 7467 & 15.9 & 2012 Sep 13 & Low & $\cdots$ \\
\hline 7596 & 16.2 & 2013 Aug 17 & Low & $\cdots$ \\
\hline 7654 & 16.1 & 2013 Aug 16 & Medium & $\cdots$ \\
\hline 7769 & 14.0 & 2012 Sep 03 & Medium & Yes \\
\hline 7914 & 14.3 & 2012 Sep 03 & Medium & Yes \\
\hline 8034 & 11.8 & 2012 Sep 03 & High & $\cdots$ \\
\hline 8227 & 10.5 & 2013 Aug 17 & High & $\cdots$ \\
\hline 8342 & 14.9 & 2012 Sep 03 & Medium & $\cdots$ \\
\hline 8405 & 15.8 & 2012 Sep 03 & Medium & $\cdots$ \\
\hline 8507 & 13.9 & 2012 Sep 03 & Medium & $\cdots$ \\
\hline 8783 & 11.5 & 2012 Sep 03 & High & $\cdots$ \\
\hline 8866 & 15.8 & 2013 Aug 16 & Low & $\cdots$ \\
\hline 9523 & 15.4 & 2013 Aug 15 & Low & $\cdots$ \\
\hline 9550 & 15.5 & 2013 Aug 19 & Low & $\cdots$ \\
\hline 9578 & 10.5 & 2013 Aug 15 & High & $\cdots$ \\
\hline 9597 & 12.0 & 2012 Sep 13 & High & $\cdots$ \\
\hline 9622 & 14.3 & 2012 Sep 04 & Medium & $\cdots$ \\
\hline 9648 & 14.9 & 2012 Sep 04 & Medium & $\cdots$ \\
\hline
\end{tabular}

Table A1

(Continued)

\begin{tabular}{|c|c|c|c|c|}
\hline NLTT & $m_{v}$ & ObsID & Obs. Qual & Companion? \\
\hline 9653 & 15.6 & 2013 Aug 16 & Low & $\cdots$ \\
\hline 9727 & 15.8 & 2013 Aug 15 & Medium & $\ldots$ \\
\hline 9734 & 15.0 & 2012 Sep 04 & Medium & $\ldots$ \\
\hline 9799 & 15.4 & 2012 Sep 13 & Low & $\cdots$ \\
\hline 9848 & 16.6 & 2013 Aug 19 & Low & $\cdots$ \\
\hline 9898 & 14.2 & 2013 Aug 19 & Low & $\ldots$ \\
\hline 9938 & 16.2 & 2013 Aug 15 & Low & $\cdots$ \\
\hline 10018 & 15.4 & 2013 Aug 17 & Low & $\ldots$ \\
\hline 10022 & 15.8 & 2013 Aug 16 & Medium & $\cdots$ \\
\hline 10135 & 15.7 & 2012 Sep 04 & Low & $\cdots$ \\
\hline 10176 & 15.8 & 2013 Aug 20 & Low & $\ldots$ \\
\hline 10243 & 14.1 & 2012 Sep 04 & Medium & $\ldots$ \\
\hline 10401 & 14.6 & 2013 Aug 18 & Low & $\ldots$ \\
\hline 10517 & 14.5 & 2012 Sep 04 & Medium & $\cdots$ \\
\hline 10536 & 11.2 & 2013 Aug 15 & High & Yes \\
\hline 10548 & 15.9 & 2013 Aug 15 & Low & $\cdots$ \\
\hline 10850 & 10.7 & 2012 Sep 04 & High & $\cdots$ \\
\hline 10883 & 15.9 & 2012 Sep 04 & Low & $\cdots$ \\
\hline 11007 & 12.2 & 2013 Aug 21 & High & $\cdots$ \\
\hline 11010 & 14.1 & 2012 Sep 04 & Medium & $\ldots$ \\
\hline 11015 & 16.3 & 2013 Aug 16 & Low & Yes \\
\hline 11032 & 14.2 & 2012 Sep 04 & Medium & $\cdots$ \\
\hline 11068 & 15.4 & 2013 Aug 21 & Low & $\cdots$ \\
\hline 11938 & 14.3 & 2012 Sep 04 & Medium & $\cdots$ \\
\hline 12017 & 12.3 & 2013 Aug 17 & High & $\cdots$ \\
\hline 12026 & 15.8 & 2013 Aug 18 & Low & $\cdots$ \\
\hline 12044 & 15.8 & 2012 Sep 13 & Low & $\cdots$ \\
\hline 12227 & 14.2 & 2013 Aug 18 & Medium & $\cdots$ \\
\hline 12350 & 12.1 & 2013 Aug 18 & Medium & $\ldots$ \\
\hline 12489 & 14.6 & 2012 Oct 10 & Low & $\ldots$ \\
\hline 12537 & 14.5 & 2013 Aug 21 & Medium & $\cdots$ \\
\hline 12704 & 15.4 & 2012 Oct 10 & Low & $\cdots$ \\
\hline 12769 & 14.1 & 2013 Aug 18 & Medium & $\cdots$ \\
\hline 12829 & 14.6 & 2012 Oct 03 & Medium & $\cdots$ \\
\hline 12845 & 10.6 & 2012 Oct 03 & High & Yes \\
\hline 12856 & 10.8 & 2013 Aug 18 & High & $\cdots$ \\
\hline 12876 & 15.6 & 2012 Oct 03 & Low & $\ldots$ \\
\hline 12923 & 15.2 & 2013 Aug 18 & Low & $\ldots$ \\
\hline 13022 & 15.9 & 2012 Oct 03 & Low & $\cdots$ \\
\hline 13344 & 13.8 & 2012 Oct 03 & Medium & $\cdots$ \\
\hline 13368 & 15.5 & 2012 Oct 03 & Low & $\cdots$ \\
\hline 13402 & 14.7 & 2012 Oct 03 & Low & $\cdots$ \\
\hline 13469 & 15.1 & 2013 Aug 18 & Low & $\ldots$ \\
\hline 13470 & 13.8 & 2012 Oct 03 & Medium & $\ldots$ \\
\hline 13641 & 12.9 & 2012 Oct 06 & High & $\cdots$ \\
\hline 13660 & 12.4 & 2012 Oct 03 & High & $\cdots$ \\
\hline 13694 & 15.4 & 2013 Aug 20 & Medium & $\cdots$ \\
\hline 13706 & 14.5 & 2012 Oct 03 & Low & $\cdots$ \\
\hline 13770 & 12.4 & 2012 Oct 03 & High & $\cdots$ \\
\hline 13811 & 13.4 & 2012 Oct 03 & Medium & $\ldots$ \\
\hline 13920 & 14.4 & 2013 Aug 20 & Medium & $\ldots$ \\
\hline 13940 & 14.4 & 2012 Oct 05 & Medium & $\ldots$ \\
\hline 14091 & 13.9 & 2012 Oct 05 & Medium & $\cdots$ \\
\hline 14131 & 13.4 & 2012 Oct 03 & Medium & $\cdots$ \\
\hline 14169 & 13.4 & 2012 Oct 05 & Medium & $\cdots$ \\
\hline 14197 & 12.4 & 2012 Oct 04 & Low & $\ldots$ \\
\hline 14391 & 13.5 & 2012 Oct 04 & Low & $\ldots$ \\
\hline 14450 & 14.7 & 2012 Oct 04 & Low & $\ldots$ \\
\hline 14549 & 14.5 & 2012 Oct 10 & Low & $\cdots$ \\
\hline 14822 & 12.7 & 2012 Oct 03 & Medium & $\cdots$ \\
\hline 14864 & 14.3 & 2012 Oct 07 & Low & $\cdots$ \\
\hline 15039 & 14.8 & 2012 Oct 10 & Low & $\cdots$ \\
\hline 15183 & 12.6 & 2012 Oct 07 & Medium & $\cdots$ \\
\hline 15218 & 12.3 & 2012 Oct 06 & High & $\cdots$ \\
\hline
\end{tabular}


Table A1

(Continued)

\begin{tabular}{|c|c|c|c|c|}
\hline NLTT & $m_{v}$ & ObsID & Obs. Qual & Companion? \\
\hline 15973 & 9.3 & 2012 Oct 07 & High & Yes \\
\hline 15974 & 13.8 & 2012 Oct 07 & High & $\ldots$ \\
\hline 16030 & 13.9 & 2012 Oct 07 & Low & $\cdots$ \\
\hline 16185 & 14.4 & 2012 Oct 10 & Low & $\cdots$ \\
\hline 16242 & 10.6 & 2012 Oct 06 & Medium & $\ldots$ \\
\hline 16579 & 12.3 & 2012 Oct 09 & High & $\ldots$ \\
\hline 16606 & 12.3 & 2012 Oct 10 & High & $\ldots$ \\
\hline 16849 & 15.3 & 2012 Oct 10 & Low & $\cdots$ \\
\hline 16869 & 13.2 & 2013 Jan 20 & High & $\ldots$ \\
\hline 16986 & 15.8 & 2013 Jan 20 & Low & $\cdots$ \\
\hline 17039 & 12.9 & 2012 Oct 10 & Medium & $\ldots$ \\
\hline 17485 & 11.9 & 2012 Oct 10 & High & Yes \\
\hline 17680 & 13.6 & 2013 Jan 20 & Medium & $\ldots$ \\
\hline 17786 & 12.0 & 2013 Jan 20 & High & $\ldots$ \\
\hline 17872 & 10.7 & 2013 Jan 20 & High & $\cdots$ \\
\hline 18019 & 13.3 & 2012 Oct 10 & Medium & $\ldots$ \\
\hline 18131 & 14.4 & 2013 Jan 20 & Medium & $\cdots$ \\
\hline 18424 & 12.7 & 2013 Jan 18 & High & $\ldots$ \\
\hline 18463 & 13.8 & 2013 Jan 20 & High & $\cdots$ \\
\hline 18502 & 12.2 & 2013 Jan 19 & High & Yes \\
\hline 18731 & 13.1 & 2013 Jan 19 & High & $\cdots$ \\
\hline 18798 & 14.5 & 2013 Jan 19 & High & Yes \\
\hline 18799 & 11.0 & 2013 Jan 19 & High & $\cdots$ \\
\hline 19037 & 14.9 & 2013 Jan 20 & Medium & $\ldots$ \\
\hline 19210 & 11.2 & 2013 Jan 20 & High & Yes \\
\hline 19301 & 14.7 & 2013 Jan 19 & Low & $\ldots$ \\
\hline 19570 & 14.4 & 2013 Apr 22 & Medium & $\cdots$ \\
\hline 19614 & 15.7 & 2013 Apr 22 & Medium & $\cdots$ \\
\hline 19643 & 11.9 & 2013 Jan 19 & High & $\cdots$ \\
\hline 19824 & 14.6 & 2013 Jan 19 & Medium & $\cdots$ \\
\hline 20252 & 14.9 & 2013 Apr 22 & Medium & $\cdots$ \\
\hline 20288 & 14.9 & 2013 Apr 22 & Medium & $\cdots$ \\
\hline 20392 & 13.8 & 2013 Jan 22 & Low & $\cdots$ \\
\hline 20476 & 13.2 & 2013 Apr 22 & High & $\ldots$ \\
\hline 20492 & 13.3 & 2013 Jan 19 & High & $\cdots$ \\
\hline 20684 & 12.0 & 2013 Jan 19 & High & $\cdots$ \\
\hline 20691 & 9.6 & 2013 Jan 19 & High & Yes \\
\hline 20768 & 14.0 & 2013 Jan 19 & Medium & $\ldots$ \\
\hline 21039 & 14.0 & 2013 Jan 19 & Medium & $\cdots$ \\
\hline 21112 & 15.3 & 2013 Apr 22 & Medium & $\cdots$ \\
\hline 21133 & 12.7 & 2013 Jan 19 & Medium & $\cdots$ \\
\hline 21341 & 14.3 & 2013 Jan 19 & Low & $\ldots$ \\
\hline 21370 & 13.7 & 2013 Jan 19 & Medium & Yes \\
\hline 21449 & 12.6 & 2013 Apr 22 & High & $\cdots$ \\
\hline 21601 & 14.6 & 2013 Apr 22 & Medium & $\cdots$ \\
\hline 22026 & 12.6 & 2013 Apr 22 & High & $\cdots$ \\
\hline 22053 & 12.1 & 2013 Jan 19 & High & $\cdots$ \\
\hline 22520 & 10.8 & 2013 Jan 19 & High & $\cdots$ \\
\hline 22752 & 13.9 & 2013 Jan 19 & Medium & $\ldots$ \\
\hline 22945 & 13.2 & 2013 Apr 22 & Medium & $\ldots$ \\
\hline 23894 & 14.6 & 2013 Jan 18 & Low & $\ldots$ \\
\hline 24006 & 15.5 & 2013 Apr 22 & Medium & $\cdots$ \\
\hline 24082 & 13.1 & 2013 Jan 19 & Medium & Yes \\
\hline 24353 & 13.2 & 2013 Jan 18 & Medium & $\cdots$ \\
\hline 24371 & 14.2 & 2013 Jan 18 & Low & $\cdots$ \\
\hline 24718 & 13.1 & 2013 Jan 18 & Medium & $\cdots$ \\
\hline 24984 & 12.5 & 2013 Apr 21 & High & $\ldots$ \\
\hline 25006 & 14.1 & 2013 Apr 21 & Medium & $\cdots$ \\
\hline 25177 & 12.2 & 2013 Apr 22 & High & $\ldots$ \\
\hline 25190 & 13.9 & 2013 Jan 18 & Low & $\cdots$ \\
\hline 25234 & 13.2 & 2013 Jan 18 & Medium & Yes \\
\hline 25475 & 13.9 & 2013 Apr 21 & Medium & $\cdots$ \\
\hline 25776 & 13.8 & 2013 Apr 22 & Medium & $\cdots$ \\
\hline 25909 & 13.5 & 2013 Apr 22 & High & $\ldots$ \\
\hline
\end{tabular}

Table A1

(Continued)

\begin{tabular}{|c|c|c|c|c|}
\hline NLTT & $m_{v}$ & ObsID & Obs. Qual & Companion? \\
\hline 25970 & 14.9 & 2013 Jan 18 & Low & $\cdots$ \\
\hline 26232 & 14.4 & 2013 Jan 18 & Low & $\ldots$ \\
\hline 26482 & 12.5 & 2013 Jan 18 & Medium & $\ldots$ \\
\hline 26503 & 14.2 & 2013 Apr 21 & Medium & $\cdots$ \\
\hline 26532 & 14.8 & 2013 Jan 18 & Low & $\cdots$ \\
\hline 26565 & 14.8 & 2013 Jan 18 & Low & $\cdots$ \\
\hline 26588 & 13.6 & 2013 Apr 21 & High & $\cdots$ \\
\hline 26677 & 13.5 & 2013 Jan 18 & Low & $\ldots$ \\
\hline 27436 & 13.0 & 2013 Jan 18 & Medium & $\ldots$ \\
\hline 27763 & 13.6 & 2013 Jan 18 & Medium & $\ldots$ \\
\hline 27767 & 14.7 & 2013 Apr 21 & Medium & $\ldots$ \\
\hline 28199 & 13.2 & 2013 Jan 18 & Medium & $\cdots$ \\
\hline 28304 & 13.3 & 2013 Apr 22 & Medium & $\ldots$ \\
\hline 28434 & 14.9 & 2013 Jan 17 & Low & Yes \\
\hline 29023 & 13.0 & 2013 Jan 18 & Medium & $\cdots$ \\
\hline 29064 & 14.0 & 2013 Apr 21 & Medium & $\cdots$ \\
\hline 29256 & 14.7 & 2013 Jan 18 & Low & $\cdots$ \\
\hline 29442 & 14.4 & 2013 Jan 18 & Low & $\ldots$ \\
\hline 29551 & 11.5 & 2013 Apr 21 & High & Yes \\
\hline 29594 & 13.2 & 2013 Apr 22 & High & Yes \\
\hline 29933 & 10.2 & 2013 Apr 22 & High & $\ldots$ \\
\hline 30128 & 13.1 & 2013 Apr 21 & High & $\cdots$ \\
\hline 30193 & 14.6 & 2013 Apr 21 & Medium & Yes \\
\hline 30462 & 12.8 & 2013 Jan 18 & Medium & $\cdots$ \\
\hline 30636 & 14.8 & 2013 Jan 18 & Low & $\cdots$ \\
\hline 30824 & 14.6 & 2013 Jan 17 & Low & $\ldots$ \\
\hline 30838 & 12.5 & 2013 Apr 22 & High & Yes \\
\hline 31146 & 12.0 & 2013 Apr 21 & High & $\cdots$ \\
\hline 31155 & 13.6 & 2013 Jan 18 & Medium & $\cdots$ \\
\hline 31240 & 15.0 & 2013 Apr 21 & Medium & Yes \\
\hline 31965 & 14.2 & 2013 Jan 19 & Medium & $\ldots$ \\
\hline 32316 & 11.3 & 2013 Apr 22 & High & $\cdots$ \\
\hline 32392 & 14.6 & 2013 Jan 19 & Medium & $\ldots$ \\
\hline 32562 & 14.3 & 2013 Jan 17 & Low & $\cdots$ \\
\hline 32648 & 12.8 & 2013 Jan 18 & Medium & $\cdots$ \\
\hline 32917 & 13.8 & 2013 Apr 22 & Medium & $\ldots$ \\
\hline 32995 & 13.4 & 2013 Apr 22 & High & $\cdots$ \\
\hline 33104 & 14.0 & 2013 Jan 18 & Low & $\cdots$ \\
\hline 33156 & 14.2 & 2013 Apr 22 & Medium & $\cdots$ \\
\hline 33371 & 12.8 & 2013 Jan 17 & Medium & $\cdots$ \\
\hline 33971 & 12.8 & 2013 Jan 18 & Medium & $\cdots$ \\
\hline 34051 & 13.5 & 2013 Jan 19 & Low & Yes \\
\hline 34628 & 11.9 & 2013 Apr 21 & High & $\ldots$ \\
\hline 35068 & 13.2 & 2013 Jan 18 & Medium & $\cdots$ \\
\hline 35318 & 13.4 & 2013 Apr 21 & High & $\cdots$ \\
\hline 36020 & 14.2 & 2013 Apr 22 & Medium & $\cdots$ \\
\hline 37342 & 14.4 & 2013 Apr 22 & High & Yes \\
\hline 37684 & 13.3 & 2013 Apr 22 & High & $\cdots$ \\
\hline 37807 & 12.0 & 2013 Apr 22 & High & $\cdots$ \\
\hline 39378 & 13.5 & 2013 Apr 22 & High & $\cdots$ \\
\hline 39721 & 13.6 & 2013 Apr 22 & High & $\cdots$ \\
\hline 40022 & 13.9 & 2013 Apr 22 & Medium & $\ldots$ \\
\hline 40313 & 13.7 & 2013 Apr 22 & High & $\ldots$ \\
\hline 41111 & 13.7 & 2013 Apr 22 & Medium & $\ldots$ \\
\hline 44039 & 11.5 & 2012 Sep 14 & High & $\cdots$ \\
\hline 44233 & 15.2 & 2012 Sep 04 & Low & $\cdots$ \\
\hline 44568 & 12.3 & 2012 Sep 04 & High & $\cdots$ \\
\hline 44639 & 11.8 & 2012 Sep 04 & High & $\cdots$ \\
\hline 44769 & 15.2 & 2013 Apr 21 & Medium & $\cdots$ \\
\hline 45609 & 12.5 & 2012 Sep 04 & High & $\ldots$ \\
\hline 45616 & 11.9 & 2012 Sep 04 & High & Yes \\
\hline 47543 & 9.2 & 2012 Oct 05 & Medium & $\ldots$ \\
\hline 48011 & 14.7 & 2012 Oct 05 & High & $\ldots$ \\
\hline 48056 & 13.7 & 2012 Oct 07 & Low & $\ldots$ \\
\hline
\end{tabular}


Table A1

(Continued)

\begin{tabular}{|c|c|c|c|c|}
\hline NLTT & $m_{v}$ & ObsID & Obs. Qual & Companion? \\
\hline 48391 & 15.2 & 2012 Oct 05 & Medium & $\cdots$ \\
\hline 48592 & 12.2 & 2012 Oct 04 & Medium & $\cdots$ \\
\hline 48866 & 12.7 & 2012 Oct 04 & Medium & $\cdots$ \\
\hline 49486 & 16.0 & 2012 Oct 04 & Medium & Yes \\
\hline 49487 & 12.3 & 2012 Oct 04 & Medium & $\ldots$ \\
\hline 49488 & 14.9 & 2013 Aug 19 & Medium & $\ldots$ \\
\hline 49618 & 12.2 & 2012 Oct 04 & Medium & $\ldots$ \\
\hline 49726 & 15.9 & 2013 Aug 19 & Low & $\cdots$ \\
\hline 49749 & 14.8 & 2012 Oct 03 & Medium & $\ldots$ \\
\hline 49819 & 14.0 & 2013 Aug 19 & High & Yes \\
\hline 49821 & 12.8 & 2013 Aug 19 & High & $\cdots$ \\
\hline 49897 & 15.8 & 2012 Oct 04 & Low & $\ldots$ \\
\hline 50257 & 13.8 & 2013 Aug 18 & Low & $\ldots$ \\
\hline 50376 & 13.9 & 2012 Sep 13 & Medium & $\ldots$ \\
\hline 50556 & 15.7 & 2012 Sep 13 & Low & $\ldots$ \\
\hline 50759 & 15.9 & 2012 Sep 13 & Low & Yes \\
\hline 50869 & 15.8 & 2013 Aug 19 & Low & $\ldots$ \\
\hline 50911 & 11.6 & 2012 Sep 13 & High & $\ldots$ \\
\hline 51006 & 14.1 & 2013 Aug 19 & Medium & Yes \\
\hline 51153 & 15.1 & 2012 Sep 13 & Low & $\cdots$ \\
\hline 51740 & 15.3 & 2012 Sep 13 & Low & $\ldots$ \\
\hline 51754 & 15.0 & 2012 Sep 13 & Low & $\ldots$ \\
\hline 51824 & 11.9 & 2013 Aug 18 & Medium & $\ldots$ \\
\hline 51856 & 13.4 & 2012 Sep 04 & Medium & $\cdots$ \\
\hline 52089 & 14.9 & 2012 Sep 04 & Medium & $\ldots$ \\
\hline 52377 & 14.5 & 2012 Sep 04 & Medium & Yes \\
\hline 52532 & 15.5 & 2012 Sep 04 & Low & Yes \\
\hline 52573 & 15.3 & 2013 Aug 18 & Low & $\ldots$ \\
\hline 52666 & 15.0 & 2013 Aug 19 & Low & $\ldots$ \\
\hline 52816 & 15.7 & 2012 Sep 13 & Low & $\cdots$ \\
\hline 52894 & 16.0 & 2012 Sep 13 & Low & $\cdots$ \\
\hline 53190 & 15.4 & 2013 Aug 16 & Medium & $\ldots$ \\
\hline 53254 & 14.7 & 2013 Aug 16 & Medium & $\cdots$ \\
\hline 53255 & 15.0 & 2013 Aug 16 & Medium & Yes \\
\hline 53274 & 11.9 & 2013 Aug 17 & High & Yes \\
\hline 53316 & 15.4 & 2012 Sep 13 & Low & $\ldots$ \\
\hline 53346 & 13.8 & 2013 Aug 17 & Medium & $\ldots$ \\
\hline 53480 & 12.6 & 2013 Aug 17 & High & $\cdots$ \\
\hline 53702 & 15.3 & 2012 Sep 13 & Medium & $\cdots$ \\
\hline 53707 & 12.1 & 2013 Aug 18 & Medium & $\ldots$ \\
\hline 53781 & 13.8 & 2013 Aug 17 & Medium & $\cdots$ \\
\hline 53801 & 11.8 & 2012 Sep 13 & High & $\cdots$ \\
\hline 53823 & 13.8 & 2013 Aug 18 & Low & $\cdots$ \\
\hline 54027 & 13.3 & 2013 Aug 19 & Medium & $\cdots$ \\
\hline 54088 & 14.1 & 2013 Aug 18 & Low & $\ldots$ \\
\hline 54168 & 13.4 & 2013 Aug 17 & Medium & $\cdots$ \\
\hline 54184 & 14.0 & 2013 Aug 17 & Medium & $\ldots$ \\
\hline 54349 & 14.4 & 2012 Sep 13 & Medium & $\cdots$ \\
\hline 54450 & 15.6 & 2013 Aug 16 & Low & $\cdots$ \\
\hline 54578 & 15.8 & 2013 Aug 18 & Low & $\ldots$ \\
\hline 54608 & 16.0 & 2013 Aug 16 & Low & $\ldots$ \\
\hline 54620 & 15.2 & 2013 Aug 17 & Medium & $\ldots$ \\
\hline 54699 & 15.1 & 2012 Sep 13 & Low & $\ldots$ \\
\hline 54710 & 15.2 & 2012 Sep 13 & Low & $\ldots$ \\
\hline 54730 & 11.5 & 2012 Sep 13 & High & $\cdots$ \\
\hline 55411 & 15.9 & 2013 Aug 16 & Low & $\cdots$ \\
\hline 55603 & 12.1 & 2013 Aug 18 & Medium & Yes \\
\hline 55732 & 13.4 & 2013 Aug 17 & Medium & $\ldots$ \\
\hline 55733 & 14.5 & 2012 Sep 03 & Medium & $\ldots$ \\
\hline 55942 & 13.5 & 2013 Aug 16 & Medium & $\ldots$ \\
\hline 56002 & 14.4 & 2012 Sep 03 & Medium & $\ldots$ \\
\hline 56290 & 12.6 & 2013 Aug 16 & High & $\ldots$ \\
\hline 56420 & 15.6 & 2012 Sep 03 & Low & $\ldots$ \\
\hline
\end{tabular}

Table A1

(Continued)

\begin{tabular}{lclcc}
\hline \hline NLTT & $m_{v}$ & ObsID & Obs. Qual & Companion? \\
\hline 56533 & 15.9 & 2013 Aug 16 & Low & $\ldots$ \\
56534 & 12.7 & 2013 Aug 17 & High & $\ldots$ \\
56774 & 12.9 & 2013 Aug 18 & Low & $\ldots$ \\
56817 & 16.1 & 2013 Aug 17 & Low & $\ldots$ \\
56818 & 14.0 & 2012 Sep 03 & Medium & Yes \\
56855 & 13.7 & 2013 Aug 16 & Medium & $\ldots$ \\
57038 & 13.9 & 2013 Aug 16 & Medium & Yes \\
57214 & 15.8 & 2013 Aug 16 & Low & $\ldots$ \\
57452 & 13.6 & 2013 Aug 16 & Medium & Yes \\
57546 & 16.2 & 2013 Aug 17 & Low & $\ldots$ \\
57564 & 10.6 & 2013 Aug 17 & High & $\ldots$ \\
57630 & 15.0 & 2013 Aug 16 & Medium & $\ldots$ \\
57631 & 13.5 & 2013 Aug 17 & Medium & $\ldots$ \\
57647 & 14.7 & 2013 Aug 17 & Medium & $\ldots$ \\
57741 & 14.2 & 2013 Aug 17 & Medium & $\ldots$ \\
57744 & 16.1 & 2013 Aug 17 & Low & $\ldots$ \\
57781 & 10.1 & 2013 Aug 16 & High & $\ldots$ \\
57832 & 15.2 & 2012 Sep 03 & Medium & $\ldots$ \\
57851 & 15.2 & 2012 Sep 03 & Medium & $\ldots$ \\
57856 & 13.2 & 2013 Aug 17 & Medium & Yes \\
58071 & 13.1 & 2012 Sep 03 & Medium & $\ldots$ \\
58141 & 15.8 & 2013 Aug 16 & Low & $\ldots$ \\
58403 & 15.2 & 2013 Aug 16 & Low & $\ldots$ \\
58522 & 15.0 & 2013 Aug 17 & Medium & $\ldots$ \\
58555 & 15.1 & 2012 Sep 03 & Medium & $\ldots$ \\
58812 & 14.9 & 2013 Aug 16 & Medium & Yes \\
\hline & & & &
\end{tabular}

thank the SOAR operators, notably Sergio Pizarro. We recognize and acknowledge the very significant cultural role and reverence that the summit of Maunakea has always had within the indigenous Hawaiian community. We are most fortunate to have the opportunity to conduct observations from this mountain. C.B. acknowledges support from the Alfred P. Sloan Foundation. This research has made use of the SIMBAD database, operated by Centre des Données Stellaires (Strasbourg, France), and bibliographic references from the Astrophysics Data System maintained by SAO/NASA.

Facilities: PO:1.5m (Robo-AO), Keck:II (NIRC2-LGS), SOAR (Goodman)

\section{APPENDIX}

In Table A1, we list our Robo-AO observed subdwarfs, including date the target was observed, observation quality as described in Section 3.1.4, and the presence of detected companions.

\section{REFERENCES}

Aarseth, S. L., \& Hills, J. G. 1972, A\&A, 21, 255

Abadi, M. G., Navarro, J. F., \& Stenmetz, M. 2006, MNRAS, 365, 747

Abell, G. O. 1959, ASPL, 8, 121

Abt, H. A. 2008, AJ, 135, 722

Abt, H. A., \& Willmarth, D. W. 1987, ApJ, 318, 786

Adams, W. S. 1915, AJ, 42, 187

Baranec, C., Riddle, R., Law, N. M., et al. 2013, J. Visualized Exp., 72, e50021

Baranec, C., Riddle, R., Law, N. M., et al. 2014, ApJL, 790, L8

Basu, S., \& Vorobyov, E. I. 2012, ApJ, 750, 30

Batten, A. H. 1973, Binary and Multiple Systems of Stars (Oxford: Pergamon)

Bourke, T. L., Myers, P. C., Evans, N. J., II, et al. 2006, ApJL, 649, L37

Bovy, J., Rix, H.-W., \& Hogg, D. W. 2012, ApJ, 751, 131 
Burgasser, A. J., Kirkpatrick, J. D., Reid, I. N., et al. 2003, ApJ, 586, 512 Carney, B. W., Laird, J. B., Latham, D. W., \& Kurucz, R. L. 1987, AJ, 94,1066

Carney, B. W., Latham, D. W., Laird, J. B., \& Anguilar, L. A. 1994, AJ, 107,2240

Chabrier, G., Baraffe, I., Allard, F., \& Hauschildt, P. 2000, ApJ, 542, 464

Chanamé, J., \& Gould, A. 2004, ApJ, 601, 289

Chiba, M., \& Beers, T. C. 2000, ApJ, 119, 2843

Clemens, J. C., Crain, J. A., \& Anderson, R. 2004, Proc. SPIE, 5492, 331

Ducati, J. R., Bevilacqua, C. M., Rembold, S. B., \& Ribeiro, D. 2001, ApJ, 558,309

Duquennoy, A., \& Mayor, M. 1991, A\&A, 248, 485

Fischer, D. A., \& Marcy, G. 1992, ApJ, 396, 178

Fischer, D. A., \& Valenti, J. 2005, ApJ, 622, 1102

Fruchter, A. S., \& Hook, R. N. 2002, PASP, 114, 144

Gizis, J. E. 1997, AJ, 113, 806

Gizis, J., \& Reid, I. N. 2000, PASP, 112, 610

Goodwin, S. P., Kroupa, P., Goodman, A., \& Burkert, A. 2007, in Protostars and Planets V, ed. B. Reipurth, D. Jewitt, \& K. Kiel (Tucson, AZ: Univ. Arizona Press), 133

Goodwin, S. P., \& Whitworth, A. 2007, A\&A, 466, 943

Gould, A. 2003, ApJ, 583, 765

Gould, A., \& Salim, S. 2003, ApJ, 582, 1001

Grether, D., \& Lineweaver, C. H. 2007, ApJ, 669, 1220

Janson, M., Bergfors, C., Brandner, W., et al. 2014, ApJS, 214, 17

Janson, M., Hormuth, F., Bergfors, C., et al. 2012, ApJ, 754, 44

Jao, W.-C., Mason, B. D., Hartkopf, W. I., et al. 2009, AJ, 137, 3800

Jaschek, C., \& Jaschek, M. 1959, Zt. F. Ap., 48, 263

Jiang, Y.-F., \& Tremaine, S. 2010, MNRAS, 401, 977

Kaib, N. A., Raymond, S. N., \& Duncan, N. 2013, Natur, 493, 381

Kaltenegger, L., \& Traub, W. A. 2009, ApJ, 609, 519

Köhler, R., Petr-Gotzens, M. G., McCaughrean, M. J., et al. 2006, A\&A, 458,461

Kopal, Z. 1959, Close Binary Systems (New York: Wiley)

Kroupa, P. 1995, MNRAS, 277, 1491

Kuiper, G. P. 1939, ApJ, 89, 548

Lafrenière, D., Marois, C., Doyon, R., Nadeau, D., \& Artigau, É 2007, ApJ, 660, 770

Latham, D. W. 2004, in ASP Conf. Ser. 318, Spectroscopically and Spatially Resolving the Components of the Close Binary Stars, ed. R. W. Hilditch, H. Hensberge, \& K. Pavlovski (San Francisco, CA: ASP), 276
Latham, D. W., Stefanik, R. P., Torres, G., et al. 2002, ApJ, 124, 1144

Law, N. M., Hodgkin, S. T., \& Mackay, C. D. 2006, MNRAS, 368, 1917

Law, N. M., Mackay, C. D., Dekany, R. G., et al. 2009, ApJ, 692, 924

Law, N. M., Morton, T., Baranec, C., et al. 2014, ApJ, 791, 35

Lépine, S., Rich, R. M., \& Shara, M. M. 2007, ApJ, 669, 1235

Lépine, S., \& Shara, M. 2005, AJ, 129, 1483

Lodieu, N., Zapatero Osorio, M. R., \& Martín, E. L. 2009, A\&A, 499, 729

López, C. E., Calandra, F., Chalela, M., et al. 2012, JDSO, 8, 78

Lutz, T. E., \& Kelker, D. H. 1973, PASP, 85, 573

Luyten, W. J. 1979, New Luyten Catalogue of Stars with Proper Motions Larger than Two-Tenths of an Arcsecond (Minneapolis, MN: Univ. Minnesota Press)

Luyten, W. J., \& Hughes, H. S. 1980, Proper-Motion Survey with the FortyEight Inch Schmidt Telescope LV First Supplement to the NLTT Catalogue (Minneapolis, MN: Univ. Minnesota Press)

Marshall, J. L. 2007, AJ, 134, 778

Marshall, J. L. 2008, AJ, 135, 1000

Meza, A., Navarro, J. F., Abadi, M. G., \& Stenmetz, M. 2005, MNRAS, 359,93

Monteiro, H., Jao, W.-C., Henry, T., et al. 2006, ApJ, 638, 446

Peter, D., Feldt, M., Henning, T., \& Hormuth, F. 2012, A\&A, 538, 74

Pourbaix, D., Tokovinin, A. A., Batten, A. H., et al. 2004, A\&A, 424, 727

Raghavan, D., McAlister, H. A., Henry, T. J., et al. 2010, ApJS, 190, 1

Reid, I. N., Brewer, C., Brucato, R. J., et al. 1991, PASP, 103, 661

Reid, I. N., \& Hawley, S. L. 2005, New Light on Dark Stars: Red Dwarfs, Low-Mass Stars, Brown Dwarfs (1st ed.; Chichester, UK: Praxis)

Riaz, B., Gizis., J., \& Samaddar, D. 2008, ApJ, 672, 1153

Riddle, R. L., Burse, M. P., Law, N. M., et al. 2012, Proc. SPIE, 8447, $84472 \mathrm{O}$

Salim, S., \& Gould, A. 2002, ApJL, 575, L83

Siegel, M. H., Majewski, S. R., Reid, I. N., \& Thompson, I. B. 2002, ApJ, 578,151

Sterzik, M. F., \& Durisen, R. H. 1998, A\&A, 339, 95

Thies, I., \& Kroupa, P. 2007, ApJ, 671, 767

Sandage, A., \& Eggen, O. 1959, MNRAS, 119, 278

van Dam, M., Bouchez, A., Le Mignant, D., et al. 2006, PASP, 118, 310

Wang, J., Fischer, D. A., Xie, J.-W., \& Ciardi, D. R. 2014, ApJ, 792, 111

Wizinowich, P., Le Mignant, D., Bouchez, A., et al. 2006, PASP, 118, 297

York, D. G., Adelman, J., Anderson, J. E., et al. 2000, AJ, 120, 1579

Zhang, Z. H., Pinfield, D. J., Burningham, B., et al. 2013, MNRAS, 434, 1005 